\title{
On Tensor Completion via Nuclear Norm Minimization
}

\author{
Ming Yuan* and Cun-Hui Zhang ${ }^{\dagger}$ \\ University of Wisconsin-Madison and Rutgers University
}

(May 9, 2014)

\begin{abstract}
Many problems can be formulated as recovering a low-rank tensor. Although an increasingly common task, tensor recovery remains a challenging problem because of the delicacy associated with the decomposition of higher order tensors. To overcome these difficulties, existing approaches often proceed by unfolding tensors into matrices and then apply techniques for matrix completion. We show here that such matricization fails to exploit the tensor structure and may lead to suboptimal procedure. More specifically, we investigate a convex optimization approach to tensor completion by directly minimizing a tensor nuclear norm and prove that this leads to an improved sample size requirement. To establish our results, we develop a series of algebraic and probabilistic techniques such as characterization of subdifferetial for tensor nuclear norm and concentration inequalities for tensor martingales, which may be of independent interests and could be useful in other tensor related problems.
\end{abstract}

${ }^{*}$ Department of Statistics, University of Wisconsin-Madison, Madison, WI 53706. The research of Ming Yuan was supported in part by NSF Career Award DMS-1321692 and FRG Grant DMS-1265202.

${ }^{\dagger}$ Department of Statistics and Biostatistics, Rutgers University, Piscataway, New Jersey 08854. The research of Cun-Hui Zhang was supported in part by NSF Grants DMS-1129626 and DMS-1209014 


\section{Introduction}

Let $\boldsymbol{T} \in \mathbb{R}^{d_{1} \times d_{2} \times \cdots \times d_{N}}$ be an $N$ th order tensor, and $\Omega$ be a randomly sampled subset of $\left[d_{1}\right] \times \cdots \times\left[d_{N}\right]$ where $[d]=\{1,2, \ldots, d\}$. The goal of tensor completion is to recover $\boldsymbol{T}$ when observing only entries $\boldsymbol{T}(\omega)$ for $\omega \in \Omega$. In particular, we are interested in the case when the dimensions $d_{1}, \ldots, d_{N}$ are large. Such a problem arises naturally in many applications. Examples include hyper-spectral image analysis ( $\mathrm{Li}$ and Li, 2010), multi-energy computed tomography (Semerci et al., 2013), radar signal processing (Sidiropoulos and Nion, 2010), audio classification (Mesgarani, Slaney and Shamma, 2006) and text mining (Cohen and Collins, 2012) among numerous others. Common to these and many other problems, the tensor $\boldsymbol{T}$ can oftentimes be identified with a certain low-rank structure. The low-rankness entails reduction in degrees of freedom, and as a result, it is possible to recover $\boldsymbol{T}$ exactly even when the sample size $|\Omega|$ is much smaller than the total number, $d_{1} d_{2} \cdots d_{N}$, of entries in $\boldsymbol{T}$.

In particular, when $N=2$, this becomes the so-called matrix completion problem which has received considerable amount of attention in recent years. See, e.g., Candès and Recht (2008), Candès and Tao (2009), Recht (2010), and Gross (2011) among many others. An especially attractive approach is through nuclear norm minimization:

$$
\min _{\boldsymbol{X} \in \mathbb{R}^{d_{1} \times d_{2}}}\|\boldsymbol{X}\|_{*} \quad \text { subject to } \boldsymbol{X}(\omega)=\boldsymbol{T}(\omega) \quad \forall \omega \in \Omega,
$$

where the nuclear norm $\|\cdot\|_{*}$ of a matrix is given by

$$
\|\boldsymbol{X}\|_{*}=\sum_{k=1}^{\min \left\{d_{1}, d_{2}\right\}} \sigma_{k}(\boldsymbol{X}),
$$

and $\sigma_{k}(\cdot)$ stands for the $k$ th largest singular value of a matrix. Denote by $\widehat{\boldsymbol{T}}$ the solution to the aforementioned nuclear norm minimization problem. As shown, for example, by Gross (2011), if an unknown $d_{1} \times d_{2}$ matrix $\boldsymbol{T}$ of rank $r$ is of low coherence with respect to the canonical basis, then it can be perfectly reconstructed by $\widehat{\boldsymbol{T}}$ with high probability whenever $|\Omega| \geq C\left(d_{1}+d_{2}\right) r \log ^{2}\left(d_{1}+d_{2}\right)$, where $C$ is a numerical constant. In other words, perfect recovery of a matrix is possible with observations from a very small fraction of entries in $\boldsymbol{T}$.

In many practical situations, we need to consider higher order tensors. The seemingly innocent task of generalizing these ideas from matrices to higher order tensor completion problems, however, turns out to be rather subtle, as basic notion such as rank, or singular value decomposition, becomes ambiguous for higher order tensors (e.g., Kolda and Bader, 2009; Hillar and Lim, 2013). A common strategy to overcome the challenges in dealing with high order tensors is to unfold them to matrices, and then resort to the usual nuclear 
norm minimization heuristics for matrices. To fix ideas, we shall focus on third order tensors $(N=3)$ in the rest of the paper although our techniques can be readily used to treat higher order tensor. Following the matricization approach, $\boldsymbol{T}$ can be reconstructed by the solution of the following convex program:

$$
\min _{\boldsymbol{X} \in \mathbb{R}^{d_{1} \times d_{2} \times d_{3}}}\left\{\left\|X^{(1)}\right\|_{*}+\left\|X^{(2)}\right\|_{*}+\left\|X^{(3)}\right\|_{*}\right\} \quad \text { subject to } \boldsymbol{X}(\omega)=\boldsymbol{T}(\omega) \quad \forall \omega \in \Omega,
$$

where $X^{(j)}$ is a $d_{j} \times\left(\prod_{k \neq j} d_{k}\right)$ matrix whose columns are the mode- $j$ fibers of $\boldsymbol{X}$. See, e.g., Liu et al. (2009), Signoretto, Lathauwer and Suykens (2010), Gandy et al. (2011), Tomioka, Hayashi and Kashima (2010), and Tomioka et al. (2011). In the light of existing results on matrix completion, with this approach, $\boldsymbol{T}$ can be reconstructed perfectly with high probability provided that

$$
|\Omega| \geq C\left(d_{1} d_{2} r_{3}+d_{1} r_{2} d_{3}+r_{1} d_{2} d_{3}\right) \log ^{2}\left(d_{1}+d_{2}+d_{3}\right)
$$

uniformly sampled entries are observed, where $r_{j}$ is the rank of $X^{(j)}$ and $C$ is a numerical constant. See, e.g., Mu et al. (2013). It is of great interests to investigate if this sample size requirement can be improved by avoiding matricization of tensors. We show here that the answer indeed is affirmative and a more direct nuclear norm minimization formulation requires a smaller sample size to recover $\boldsymbol{T}$.

More specifically, write, for two tensors $\boldsymbol{X}, \boldsymbol{Y} \in \mathbb{R}^{d_{1} \times d_{2} \times d_{3}}$,

$$
\langle\boldsymbol{X}, \boldsymbol{Y}\rangle=\sum_{\omega \in\left[d_{1}\right] \times\left[d_{2}\right] \times\left[d_{3}\right]} \boldsymbol{X}(\omega) \boldsymbol{Y}(\omega)
$$

as their inner product. Define

$$
\|\boldsymbol{X}\|=\max _{\boldsymbol{u}_{j} \in \mathbb{R}^{d_{j}}:\left\|\boldsymbol{u}_{1}\right\|=\left\|\boldsymbol{u}_{2}\right\|=\left\|\boldsymbol{u}_{3}\right\|=1}\left\langle\boldsymbol{X}, \boldsymbol{u}_{1} \otimes \boldsymbol{u}_{2} \otimes \boldsymbol{u}_{3}\right\rangle
$$

where, with slight abuse of notation, $\|\cdot\|$ also stands for the usual Euclidean norm for a vector, and for vectors $\boldsymbol{u}_{j}=\left(u_{1}^{j}, \ldots, u_{d_{j}}^{j}\right)^{\top}$,

$$
\boldsymbol{u}_{1} \otimes \boldsymbol{u}_{2} \otimes \boldsymbol{u}_{3}=\left(u_{a}^{1} u_{b}^{2} u_{c}^{3}\right)_{1 \leq a \leq d_{1}, 1 \leq b \leq d_{2}, 1 \leq c \leq d_{3}} .
$$

It is clear that the $\|\cdot\|$ defined above for tensors is a norm and can be viewed as an extension of the usual matrix spectral norm. Appealing to the duality between the spectral norm and nuclear norm in the matrix case, we now consider the following nuclear norm for tensors:

$$
\|\boldsymbol{X}\|_{*}=\max _{\boldsymbol{Y} \in \mathbb{R}^{d_{1} \times d_{2} \times d_{3}:\|\boldsymbol{Y}\| \leq 1}}\langle\boldsymbol{Y}, \boldsymbol{X}\rangle
$$


It is clear that $\|\cdot\|_{*}$ is also a norm. We then consider reconstructing $\boldsymbol{T}$ via the solution to the following convex program:

$$
\min _{\boldsymbol{X} \in \mathbb{R}^{d_{1} \times d_{2} \times d_{3}}}\|\boldsymbol{X}\|_{*} \quad \text { subject to } \boldsymbol{X}(\omega)=\boldsymbol{T}(\omega) \quad \forall \omega \in \Omega \text {. }
$$

We show that the sample size requirement for perfect recovery of a tensor with low coherence using this approach is

$$
|\Omega| \geq C\left(r^{2}\left(d_{1}+d_{2}+d_{3}\right)+\sqrt{r d_{1} d_{2} d_{3}}\right) \text { polylog }\left(d_{1}+d_{2}+d_{3}\right),
$$

where

$$
r=\sqrt{\left(r_{1} r_{2} d_{3}+r_{1} r_{3} d_{2}+r_{2} r_{3} d_{1}\right) /\left(d_{1}+d_{2}+d_{3}\right)}
$$

polylog $(x)$ is a certain polynomial function of $\log (x)$, and $C$ is a numerical constant. In particular, when considering (nearly) cubic tensors with $d_{1}, d_{2}$ and $d_{3}$ approximately equal to a common $d$, then this sample size requirement is essentially of the order $r^{1 / 2}(d \log d)^{3 / 2}$. In the case when the tensor dimension $d$ is large while the rank $r$ is relatively small, this can be a drastic improvement over the existing results based on matricizing tensors where the sample size requirement is $r(d \log d)^{2}$.

The high-level strategy to the investigation of the proposed nuclear norm minimization approach for tensors is similar, in a sense, to the treatment of matrix completion. Yet the analysis for tensors is much more delicate and poses significant new challenges because many of the well-established tools for matrices, either algebraic such as characterization of the subdifferential of the nuclear norm, or probabilistic such as concentration inequalities for martingales, do not exist for tensors. Some of these disparities can be bridged and we develop various tools to do so. Others are due to fundamental differences between matrices and higher order tensors, and we devise new strategies to overcome them. The tools and techniques we developed may be of independent interests and can be useful in dealing with other problems for tensors.

The rest of the paper is organized as follows. We first describe some basic properties of tensors and their nuclear norm necessary for our analysis in Section 2, Section 3 discusses the main architect of our analysis. The main probabilistic tools we use are concentration bounds for the sum of random tensors. Because the tensor spectral norm does not have the interpretation as an operator norm of a linear mapping between Hilbert spaces, the usual matrix Bernstein inequality cannot be directly applied. It turns out that different strategies are required for tensors of low rank and tensors with sparse support, and these results are presented in Sections 4 and 5 respectively. We conclude the paper with a few remarks in Section 6 , 


\section{Tensor}

We first collect some useful algebraic facts for tensors essential to our later analysis. Recall that the inner product between two third order tensors $\boldsymbol{X}, \boldsymbol{Y} \in \mathbb{R}^{d_{1} \times d_{2} \times d_{3}}$ is given by

$$
\langle\boldsymbol{X}, \boldsymbol{Y}\rangle=\sum_{a=1}^{d_{1}} \sum_{b=1}^{d_{2}} \sum_{c=1}^{d_{3}} \boldsymbol{X}(a, b, c) \boldsymbol{Y}(a, b, c),
$$

and $\|\boldsymbol{X}\|_{\text {HS }}=\langle\boldsymbol{X}, \boldsymbol{X}\rangle^{1 / 2}$ is the usual Hilbert-Schmidt norm of $\boldsymbol{X}$. Another tensor norm of interest is the entrywise $\ell_{\infty}$ norm, or tensor max norm:

$$
\|\boldsymbol{X}\|_{\max }=\max _{\omega \in\left[d_{1}\right] \times\left[d_{2}\right] \times\left[d_{3}\right]}|\boldsymbol{X}(\omega)| .
$$

It is clear that for any the third order tensor $\boldsymbol{X} \in \mathbb{R}^{d_{1} \times d_{2} \times d_{3}}$,

$$
\|\boldsymbol{X}\|_{\max } \leq\|\boldsymbol{X}\| \leq\|\boldsymbol{X}\|_{\mathrm{HS}} \leq\|\boldsymbol{X}\|_{*}, \quad \text { and } \quad\|\boldsymbol{X}\|_{\mathrm{HS}}^{2} \leq\|\boldsymbol{X}\|_{*}\|\boldsymbol{X}\|
$$

We shall also encounter linear maps defined on tensors. Let $\mathcal{R}: \mathbb{R}^{d_{1} \times d_{2} \times d_{3}} \rightarrow \mathbb{R}^{d_{1} \times d_{2} \times d_{3}}$ be a linear map. We define the induced operator norm of $\mathcal{R}$ under tensor Hilbert-Schmidt norm as

$$
\|\mathcal{R}\|=\max \left\{\|\mathcal{R} \boldsymbol{X}\|_{\mathrm{HS}}: \boldsymbol{X} \in \mathbb{R}^{d_{1} \times d_{2} \times d_{3}},\|\boldsymbol{X}\|_{\mathrm{HS}} \leq 1\right\}
$$

\subsection{Decomposition and Projection}

Consider the following tensor decomposition of $\boldsymbol{X}$ into rank-one tensors:

$$
\boldsymbol{X}=[A, B, C]:=\sum_{k=1}^{r} \boldsymbol{a}_{k} \otimes \boldsymbol{b}_{k} \otimes \boldsymbol{c}_{k},
$$

where $\boldsymbol{a}_{k} \mathrm{~s}, \boldsymbol{b}_{k} \mathrm{~s}$ and $\boldsymbol{c}_{k} \mathrm{~s}$ are the column vectors of matrices $A, B$ and $C$ respectively. Such a decomposition in general is not unique (see, e.g., Kruskal, 1989). However, the linear spaces spanned by columns of $A, B$ and $C$ respectively are uniquely defined.

More specifically, write $\boldsymbol{X}(\cdot, b, c)=\left(\boldsymbol{X}(1, b, c), \ldots, \boldsymbol{X}\left(d_{1}, b, c\right)\right)^{\top}$, that is the mode-1 fiber of $\boldsymbol{X}$. Define $\boldsymbol{X}(a, \cdot, c)$ and $\boldsymbol{X}(a, b, \cdot)$ in a similar fashion. Let

$$
\begin{aligned}
& \mathcal{L}_{1}(\boldsymbol{X})=\text { l.s. }\left\{\boldsymbol{X}(\cdot, b, c): 1 \leq b \leq d_{2}, 1 \leq c \leq d_{3}\right\} \\
& \mathcal{L}_{2}(\boldsymbol{X})=\text { l.s. }\left\{\boldsymbol{X}(a, \cdot, c): 1 \leq a \leq d_{1}, 1 \leq c \leq d_{3}\right\} \\
& \mathcal{L}_{3}(\boldsymbol{X})=\text { l.s. }\left\{\boldsymbol{X}(a, b, \cdot): 1 \leq a \leq d_{1}, 1 \leq b \leq d_{2}\right\}
\end{aligned}
$$

where 1.s. represents the linear space spanned by a collection of vectors of conformable dimension. Then it is clear that the linear space spanned by the column vectors of $A$ is 
$\mathcal{L}_{1}(\boldsymbol{X})$, and similar statements hold true for the column vectors of $B$ and $C$. In the case of matrices, both marginal linear spaces, $\mathcal{L}_{1}$ and $\mathcal{L}_{2}$ are necessarily of the same dimension as they are spanned by the respective singular vectors. For higher order tensors, however, this is typically not true. We shall denote by $r_{j}(\boldsymbol{X})$ the dimension of $\mathcal{L}_{j}(\boldsymbol{X})$ for $j=1,2$ and 3 , which are often referred to the Tucker ranks of $\boldsymbol{X}$. Another useful notion of "tensor rank" for our purposes is

$$
\bar{r}(\boldsymbol{X})=\sqrt{\left(r_{1}(\boldsymbol{X}) r_{2}(\boldsymbol{X}) d_{3}+r_{1}(\boldsymbol{X}) r_{3}(\boldsymbol{X}) d_{2}+r_{2}(\boldsymbol{X}) r_{3}(\boldsymbol{X}) d_{1}\right) / d}
$$

where $d=d_{1}+d_{2}+d_{3}$, which can also be viewed as a generalization of the matrix rank to tensors. It is well known that the smallest value for $r$ in the rank-one decomposition (11) is in $\left[\bar{r}(\boldsymbol{X}), \bar{r}^{2}(\boldsymbol{X})\right]$.

Let $M$ be a matrix of size $d_{0} \times d_{1}$. Marginal multiplication of $M$ and a tensor $\boldsymbol{X}$ in the first coordinate yields a tensor of size $d_{0} \times d_{2} \times d_{3}$ :

$$
\left(M \times_{1} \boldsymbol{X}\right)(a, b, c)=\sum_{a^{\prime}=1}^{d_{1}} M_{a a^{\prime}} X\left(a^{\prime}, b, c\right) .
$$

It is easy to see that if $\boldsymbol{X}=[A, B, C]$, then $M \times{ }_{1} \boldsymbol{X}=[M A, B, C]$. Marginal multiplications $\times_{2}$ and $\times_{3}$ between a matrix of conformable size and $\boldsymbol{X}$ can be similarly defined.

Let $\boldsymbol{P}$ be arbitrary projection from $\mathbb{R}^{d_{1}}$ to a linear subspace of $\mathbb{R}^{d_{1}}$. It is clear from the definition of marginal multiplications, $[\boldsymbol{P} A, B, C]$ is also uniquely defined for tensor $\boldsymbol{X}=[A, B, C]$, that is, $[\boldsymbol{P} A, B, C]$ does not depend on the particular decomposition of $A$, $B, C$. Now let $\boldsymbol{P}_{j}$ be arbitrary projection from $\mathbb{R}^{d_{j}}$ to a linear subspace of $\mathbb{R}^{d_{j}}$. Define a tensor projection $\boldsymbol{P}_{1} \otimes \boldsymbol{P}_{2} \otimes \boldsymbol{P}_{3}$ on $\boldsymbol{X}=[A, B, C]$ as

$$
\left(\boldsymbol{P}_{1} \otimes \boldsymbol{P}_{2} \otimes \boldsymbol{P}_{3}\right) \boldsymbol{X}=\left[\boldsymbol{P}_{1} A, \boldsymbol{P}_{2} B, \boldsymbol{P}_{3} C\right]
$$

We note that there is no ambiguity in defining $\left(\boldsymbol{P}_{1} \otimes \boldsymbol{P}_{2} \otimes \boldsymbol{P}_{3}\right) \boldsymbol{X}$ because of the uniqueness of marginal projections.

Recall that $\mathcal{L}_{j}(\boldsymbol{X})$ is the linear space spanned by the mode- $j$ fibers of $\boldsymbol{X}$. Let $\boldsymbol{P}_{\boldsymbol{X}}^{j}$ be the projection from $\mathbb{R}^{d_{j}}$ to $\mathcal{L}_{j}(\boldsymbol{X})$, and $\boldsymbol{P}_{\boldsymbol{X}^{\perp}}^{j}$ be the projection to its orthogonal complement in $\mathbb{R}^{d_{j}}$. The following tensor projections will be used extensively in our analysis:

$$
\begin{array}{cl}
\mathcal{Q}_{\boldsymbol{X}}^{0}=\boldsymbol{P}_{\boldsymbol{X}}^{1} \otimes \boldsymbol{P}_{\boldsymbol{X}}^{2} \otimes \boldsymbol{P}_{\boldsymbol{X}}^{3}, & \mathcal{Q}_{\boldsymbol{X}^{\perp}}^{0}=\boldsymbol{P}_{\boldsymbol{X}^{\perp}}^{1} \otimes \boldsymbol{P}_{\boldsymbol{X}^{\perp}}^{2} \otimes \boldsymbol{P}_{\boldsymbol{X}^{\perp}}^{3}, \\
\mathcal{Q}_{\boldsymbol{X}}^{1}=\boldsymbol{P}_{\boldsymbol{X}^{\perp}}^{1} \otimes \boldsymbol{P}_{\boldsymbol{X}}^{2} \otimes \boldsymbol{P}_{\boldsymbol{X}}^{3}, & \mathcal{Q}_{\boldsymbol{X}^{\perp}}^{1}=\boldsymbol{P}_{\boldsymbol{X}}^{1} \otimes \boldsymbol{P}_{\boldsymbol{X}^{\perp}}^{2} \otimes \boldsymbol{P}_{\boldsymbol{X}^{\perp}}^{3}, \\
\mathcal{Q}_{\boldsymbol{X}}^{2}=\boldsymbol{P}_{\boldsymbol{X}}^{1} \otimes \boldsymbol{P}_{\boldsymbol{X}^{\perp}}^{2} \otimes \boldsymbol{P}_{\boldsymbol{X}}^{3}, & \mathcal{Q}_{\boldsymbol{X}^{\perp}}^{2}=\boldsymbol{P}_{\boldsymbol{X}^{\perp}}^{1} \otimes \boldsymbol{P}_{\boldsymbol{X}}^{2} \otimes \boldsymbol{P}_{\boldsymbol{X}^{\perp}}^{3}, \\
\mathcal{Q}_{\boldsymbol{X}}^{3}=\boldsymbol{P}_{\boldsymbol{X}}^{1} \otimes \boldsymbol{P}_{\boldsymbol{X}}^{2} \otimes \boldsymbol{P}_{\boldsymbol{X}^{\perp}}^{3}, & \mathcal{Q}_{\boldsymbol{X}^{\perp}}^{3}=\boldsymbol{P}_{\boldsymbol{X}^{\perp}}^{1} \otimes \boldsymbol{P}_{\boldsymbol{X}^{\perp}}^{2} \otimes \boldsymbol{P}_{\boldsymbol{X}}^{3}, \\
\mathcal{Q}_{\boldsymbol{X}}=\mathcal{Q}_{\boldsymbol{X}}^{0}+\mathcal{Q}_{\boldsymbol{X}}^{1}+\mathcal{Q}_{\boldsymbol{X}}^{2}+\mathcal{Q}_{\boldsymbol{X}}^{3}, & \mathcal{Q}_{\boldsymbol{X}^{\perp}}=\mathcal{Q}_{\boldsymbol{X}^{\perp}}^{0}+\mathcal{Q}_{\boldsymbol{X}^{\perp}}^{1}+\mathcal{Q}_{\boldsymbol{X}^{\perp}}^{2}+\mathcal{Q}_{\boldsymbol{X}^{\perp}}^{3}
\end{array}
$$




\subsection{Subdifferential of Tensor Nuclear Norm}

One of the main technical tools in analyzing the nuclear norm minimization is the characterization of the subdifferntial of the nuclear norm. Such results are well known in the case of matrices. In particular, let $M=U D V^{\top}$ be the singular value decomposition of a matrix $M$, then the subdifferential of the nuclear norm at $M$ is given by

$$
\partial\|\cdot\|_{*}(M)=\left\{U V^{\top}+W: U^{\top} W=W^{\top} V=0, \quad \text { and } \quad\|W\| \leq 1\right\},
$$

where with slight abuse of notion, $\|\cdot\|_{*}$ and $\|\cdot\|$ are the nuclear and spectral norms of matrices. See, e.g., Watson (1992). In other words, for any other matrix $Y$ of conformable dimensions,

$$
\|Y\|_{*} \geq\|M\|_{*}+\left\langle U V^{\top}+W, Y-M\right\rangle
$$

if and only if $U^{\top} W=W^{\top} V=0$ and $\|W\| \leq 1$. Characterizing the subdifferential of the nuclear norm for higher order tensors is more subtle due to the lack of corresponding spectral decomposition.

A straightforward generalization of the above characterization may suggest that $\partial\|\cdot\|_{*}(\boldsymbol{X})$ be identified with

$$
\left\{\boldsymbol{W}+\boldsymbol{W}^{\perp}: \boldsymbol{W}^{\perp}=\mathcal{Q}_{\boldsymbol{X}^{\perp}} \boldsymbol{W}^{\perp},\left\|\boldsymbol{W}^{\perp}\right\| \leq 1\right\}
$$

for some $\boldsymbol{W}$ in the range of $\mathcal{Q}_{\boldsymbol{X}}^{0}$. It turns out that this in general is not true. As a simple counterexample, let

$$
\boldsymbol{X}=\boldsymbol{e}_{1} \otimes \boldsymbol{e}_{1} \otimes \boldsymbol{e}_{1}
$$

and

$$
\boldsymbol{Y}=\sum_{1 \leq i, j, k \leq 2} \boldsymbol{e}_{i} \otimes \boldsymbol{e}_{j} \otimes \boldsymbol{e}_{k}=\left(\boldsymbol{e}_{1}+\boldsymbol{e}_{2}\right) \otimes\left(\boldsymbol{e}_{1}+\boldsymbol{e}_{2}\right) \otimes\left(\boldsymbol{e}_{1}+\boldsymbol{e}_{2}\right),
$$

where $d_{1}=d_{2}=d_{3}=2$ and $\boldsymbol{e}_{i}$ 's are the canonical basis of an Euclidean space. It is clear that $\|\boldsymbol{X}\|_{*}=1$ and $\|\boldsymbol{Y}\|_{*}=2 \sqrt{2}$. Take $\boldsymbol{W}^{\perp}=\boldsymbol{U} /\|\boldsymbol{U}\|$ where

$$
\boldsymbol{U}=\boldsymbol{e}_{1} \otimes \boldsymbol{e}_{2} \otimes \boldsymbol{e}_{2}+\boldsymbol{e}_{2} \otimes \boldsymbol{e}_{1} \otimes \boldsymbol{e}_{2}+\boldsymbol{e}_{2} \otimes \boldsymbol{e}_{2} \otimes \boldsymbol{e}_{1}
$$

As we shall show in the proof of Lemma 1 below, $\|\boldsymbol{U}\|=2 / \sqrt{3}$. It is clear that $\boldsymbol{W}^{\perp}=$ $\mathcal{Q}_{\boldsymbol{X}^{\perp}} \boldsymbol{W}^{\perp}$ and $\left\|\boldsymbol{W}^{\perp}\right\| \leq 1$. Yet,

$$
\|\boldsymbol{X}\|_{*}+\left\langle\boldsymbol{Y}-\boldsymbol{X}, \boldsymbol{W}+\boldsymbol{W}^{\perp}\right\rangle=1+\left\langle\boldsymbol{Y}-\boldsymbol{X}, \boldsymbol{W}^{\perp}\right\rangle=1+3 \sqrt{3} / 2>2 \sqrt{2}=\|\boldsymbol{Y}\|_{*},
$$

for any $\boldsymbol{W}$ such that $\boldsymbol{W}=\mathcal{Q}_{\boldsymbol{X}}^{0} \boldsymbol{W}$.

Fortunately, for our purposes, the following relaxed characterization is sufficient. 
Lemma 1 For any third order tensor $\boldsymbol{X} \in \mathbb{R}^{d_{1} \times d_{2} \times d_{3}}$, there exists a $\boldsymbol{W} \in \mathbb{R}^{d_{1} \times d_{2} \times d_{3}}$ such that $\boldsymbol{W}=\mathcal{Q}_{X}^{0} \boldsymbol{W},\|\boldsymbol{W}\|=1$ and

$$
\|\boldsymbol{X}\|_{*}=\langle\boldsymbol{W}, \boldsymbol{X}\rangle
$$

Furthermore, for any $\boldsymbol{Y} \in \mathbb{R}^{d_{1} \times d_{2} \times d_{3}}$ and $\boldsymbol{W}^{\perp} \in \mathbb{R}^{d_{1} \times d_{2} \times d_{3}}$ obeying $\left\|\boldsymbol{W}^{\perp}\right\| \leq 1 / 2$,

$$
\|\boldsymbol{Y}\|_{*} \geq\|\boldsymbol{X}\|_{*}+\left\langle\boldsymbol{W}+\mathcal{Q}_{\boldsymbol{X}^{\perp}} \boldsymbol{W}^{\perp}, \boldsymbol{Y}-\boldsymbol{X}\right\rangle
$$

Proof of Lemma [1. If $\|\boldsymbol{W}\| \leq 1$, then

$$
\max _{\left\|\boldsymbol{u}_{j}\right\|=1}\left\langle\mathcal{Q}_{\boldsymbol{X}}^{0} \boldsymbol{W}, \boldsymbol{u}_{1} \otimes \boldsymbol{u}_{2} \otimes \boldsymbol{u}_{3}\right\rangle \leq \max _{\left\|\boldsymbol{u}_{j}\right\|=1}\left\langle\boldsymbol{W},\left(\boldsymbol{P}_{\boldsymbol{X}}^{1} \boldsymbol{u}_{1}\right) \otimes\left(\boldsymbol{P}_{\boldsymbol{X}}^{2} \boldsymbol{u}_{2}\right) \otimes\left(\boldsymbol{P}_{\boldsymbol{X}}^{3} \boldsymbol{u}_{3}\right)\right\rangle \leq 1
$$

It follows that

$$
\|\boldsymbol{X}\|_{*}=\max _{\|\boldsymbol{W}\|=1}\langle\boldsymbol{W}, \boldsymbol{X}\rangle=\max _{\|\boldsymbol{W}\|=1}\left\langle\mathcal{Q}_{\boldsymbol{X}}^{0} \boldsymbol{W}, \boldsymbol{X}\right\rangle
$$

is attained with a certain $\boldsymbol{W}$ satisfying $\|\boldsymbol{W}\|=\left\|\mathcal{Q}_{\boldsymbol{X}}^{0} \boldsymbol{W}\right\|=1$.

Now consider a tensor $\boldsymbol{W}^{\perp}$ satisfying

$$
\left\|\boldsymbol{W}+\mathcal{Q}_{\boldsymbol{X}^{\perp}} \boldsymbol{W}^{\perp}\right\| \leq 1
$$

Because $\mathcal{Q}_{\boldsymbol{X}^{\perp}} \boldsymbol{X}=0$, it follows from the definition of the tensor nuclear norm that

$$
\left\langle\boldsymbol{W}+\mathcal{Q}_{\boldsymbol{X}^{\perp}} \boldsymbol{W}^{\perp}, \boldsymbol{Y}-\boldsymbol{X}\right\rangle \leq\|\boldsymbol{Y}\|_{*}-\langle\boldsymbol{W}, \boldsymbol{X}\rangle=\|\boldsymbol{Y}\|_{*}-\|\boldsymbol{X}\|_{*}
$$

It remains to prove that $\left\|\boldsymbol{W}^{\perp}\right\| \leq 1 / 2$ implies

$$
\left\|\boldsymbol{W}+\mathcal{Q}_{\boldsymbol{X}^{\perp}} \boldsymbol{W}^{\perp}\right\| \leq 1 .
$$

Recall that $\boldsymbol{W}=\mathcal{Q}_{\boldsymbol{X}}^{0} \boldsymbol{W},\left\|\boldsymbol{W}^{\perp}\right\| \leq 1 / 2$, and $\left\|\boldsymbol{u}_{j}\right\|=1$. Then

$$
\begin{aligned}
& \left\langle\boldsymbol{W}+\mathcal{Q}_{\boldsymbol{X}^{\perp}} \boldsymbol{W}^{\perp}, \boldsymbol{u}_{1} \otimes \boldsymbol{u}_{2} \otimes \boldsymbol{u}_{3}\right\rangle \\
\leq & \left\|\mathcal{Q}_{\boldsymbol{X}}^{0}\left(\boldsymbol{u}_{1} \otimes \boldsymbol{u}_{2} \otimes \boldsymbol{u}_{3}\right)\right\|_{*}+\frac{1}{2}\left\|\mathcal{Q}_{\boldsymbol{X}^{\perp}}\left(\boldsymbol{u}_{1} \otimes \boldsymbol{u}_{2} \otimes \boldsymbol{u}_{3}\right)\right\|_{*} \\
\leq & \prod_{j=1}^{3} \sqrt{1-a_{j}^{2}}+\frac{1}{2}\left(a_{1} a_{2}+a_{1} a_{3} \sqrt{1-a_{2}^{2}}+a_{2} a_{3} \sqrt{1-a_{1}^{2}}\right) .
\end{aligned}
$$

where $a_{j}=\left\|\boldsymbol{P}_{\boldsymbol{X}^{\perp}}^{j} \boldsymbol{u}_{j}\right\|_{2}$, for $j=1,2,3$. Let $x=a_{1} a_{2}$ and

$$
y=\sqrt{\left(1-a_{1}^{2}\right)\left(1-a_{2}^{2}\right)}
$$


We have

$$
\begin{aligned}
\left(a_{1} \sqrt{1-a_{2}^{2}}+a_{2} \sqrt{1-a_{1}^{2}}\right)^{2} & =a_{1}^{2}\left(1-a_{2}^{2}\right)+a_{2}^{2}\left(1-a_{1}^{2}\right)+2 x y \\
& =a_{1}^{2}+a_{2}^{2}-2 a_{1}^{2} a_{2}^{2}+2 x y \\
& =1-(y-x)^{2} .
\end{aligned}
$$

It follows that for any value of $a_{3} \in(0,1)$,

$$
\left\langle\boldsymbol{W}+\mathcal{Q}_{\boldsymbol{X}^{\perp}} \boldsymbol{W}^{\perp}, \boldsymbol{u}_{1} \otimes \boldsymbol{u}_{2} \otimes \boldsymbol{u}_{3}\right\rangle \leq y \sqrt{1-a_{3}^{2}}+\frac{1}{2}\left(x+a_{3} \sqrt{1-(y-x)^{2}}\right) .
$$

This function of $(x, y)$ is increasing in the smaller of $x$ and $y$. For $x<y$, the maximum of $x^{2}$ given $y^{2}$ is attained when $a_{1}=a_{2}$ by simple calculation with the Lagrange multiplier. Similarly, for $y<x$, the maximum of $y^{2}$ given $x^{2}$ is attained when $a_{1}=a_{2}$. Thus, setting $a_{1}=a_{2}=a$, we find

$$
\left\langle\boldsymbol{W}+\mathcal{Q}_{\boldsymbol{X}^{\perp}} \boldsymbol{W}^{\perp}, \boldsymbol{u}_{1} \otimes \boldsymbol{u}_{2} \otimes \boldsymbol{u}_{3}\right\rangle \leq \max _{a_{3}, a}\left\{\left(1-a^{2}\right) \sqrt{1-a_{3}^{2}}+\frac{1}{2}\left(a^{2}+2 a_{3} a \sqrt{1-a^{2}}\right)\right\} .
$$

The above maximum is attained when $a_{3}=a$. Because $\sqrt{1-a^{2}}+a^{2} / 2 \leq 1$, we have

$$
\left\langle\boldsymbol{W}+\mathcal{Q}_{\boldsymbol{X}^{\perp}} \boldsymbol{W}^{\perp}, \boldsymbol{u}_{1} \otimes \boldsymbol{u}_{2} \otimes \boldsymbol{u}_{3}\right\rangle \leq 1,
$$

which completes the proof of the lemma.

The norm of $\boldsymbol{U}$ defined in (2) can be computed using a similar argument:

$$
\begin{aligned}
\|\boldsymbol{U}\| & =\max _{0 \leq a_{1} \leq a_{2} \leq a_{3} \leq 1}\left(a_{1} a_{2} \sqrt{1-a_{3}^{2}}+a_{1} a_{3} \sqrt{1-a_{2}^{2}}+a_{2} a_{3} \sqrt{1-a_{1}^{2}}\right) \\
& =\max _{x, y, a_{3}}\left(x \sqrt{1-a_{3}^{2}}+a_{3} \sqrt{1-(y-x)^{2}}\right) \\
& =\max _{a, a_{3}} a\left(a \sqrt{1-a_{3}^{2}}+2 a_{3} \sqrt{1-a^{2}}\right), \\
& =\max _{a} a \sqrt{a^{2}+4\left(1-a^{2}\right)},
\end{aligned}
$$

which yields $\|\boldsymbol{U}\|=2 / \sqrt{3}$ with $a^{2}=2 / 3$.

Note that Lemma 1 gives only sufficient conditions of the subgradient of tensor nuclear norm. Equivalently it states that

$$
\partial\|\cdot\|_{*}(\boldsymbol{X}) \supseteq\left\{\boldsymbol{W}+\mathcal{Q}_{\boldsymbol{X}^{\perp}} \boldsymbol{W}^{\perp}:\left\|\boldsymbol{W}^{\perp}\right\| \leq 1 / 2\right\} .
$$

The main difference between the above characterization and that for the usual matrix nuclear norm is the requirement that $\left\|\boldsymbol{W}^{\perp}\right\|$ is no greater than $1 / 2$ instead of 1 . We note also that the constant $1 / 2$ may be further improved. No attempt has been made here to sharpen the constant as it already suffices for our analysis. 


\subsection{Coherence}

A central concept to matrix completion is coherence. Recall that the coherence of an $r$ dimensional linear subspace $U$ of $\mathbb{R}^{k}$ is defined as

$$
\mu(U)=\frac{k}{r} \max _{1 \leq i \leq k}\left\|\boldsymbol{P}_{U} \boldsymbol{e}_{i}\right\|^{2}=\frac{\max _{1 \leq i \leq k}\left\|\boldsymbol{P}_{U} \boldsymbol{e}_{i}\right\|^{2}}{k^{-1} \sum_{i=1}^{k}\left\|\boldsymbol{P}_{U} \boldsymbol{e}_{i}\right\|^{2}},
$$

where $\boldsymbol{P}_{U}$ is the orthogonal projection onto $U$ and $\boldsymbol{e}_{i}$ 's are the canonical basis for $\mathbb{R}^{k}$. See, e.g., Candès and Recht (2008). We shall define the coherence of a tensor $\boldsymbol{X} \in \mathbb{R}^{d_{1} \times d_{2} \times d_{3}}$ as

$$
\mu(\boldsymbol{X})=\max \left\{\mu\left(\mathcal{L}_{1}(\boldsymbol{X})\right), \mu\left(\mathcal{L}_{2}(\boldsymbol{X})\right), \mu\left(\mathcal{L}_{3}(\boldsymbol{X})\right)\right\} .
$$

It is clear that $\mu(\boldsymbol{X}) \geq 1$, since $\mu(U)$ is the ratio of the $\ell_{\infty}$ and length-normalized $\ell_{2}$ norms of a vector.

Lemma 2 Let $\boldsymbol{X} \in \mathbb{R}^{d_{1} \times d_{2} \times d_{3}}$ be a third order tensor. Then

$$
\max _{a, b, c}\left\|\mathcal{Q}_{\boldsymbol{X}}\left(\boldsymbol{e}_{a} \otimes \boldsymbol{e}_{b} \otimes \boldsymbol{e}_{c}\right)\right\|_{\mathrm{HS}}^{2} \leq \frac{\bar{r}^{2}(\boldsymbol{X}) d}{d_{1} d_{2} d_{3}} \mu^{2}(\boldsymbol{X}) .
$$

Proof of Lemma 2, Recall that $\mathcal{Q}_{\boldsymbol{X}}=\mathcal{Q}_{\boldsymbol{X}}^{0}+\mathcal{Q}_{\boldsymbol{X}}^{1}+\mathcal{Q}_{\boldsymbol{X}}^{2}+\mathcal{Q}_{\boldsymbol{X}}^{3}$. Therefore,

$$
\begin{aligned}
\left\|\mathcal{Q}_{\boldsymbol{X}}\left(\boldsymbol{e}_{a} \otimes \boldsymbol{e}_{b} \otimes \boldsymbol{e}_{c}\right)\right\|^{2}= & \sum_{j, k=0}^{3}\left\langle\mathcal{Q}_{\boldsymbol{X}}^{j}\left(\boldsymbol{e}_{a} \otimes \boldsymbol{e}_{b} \otimes \boldsymbol{e}_{c}\right), \mathcal{Q}_{\boldsymbol{X}}^{k}\left(\boldsymbol{e}_{a} \otimes \boldsymbol{e}_{b} \otimes \boldsymbol{e}_{c}\right)\right\rangle \\
= & \sum_{j=0}^{3}\left\|\mathcal{Q}_{\boldsymbol{X}}^{j}\left(\boldsymbol{e}_{a} \otimes \boldsymbol{e}_{b} \otimes \boldsymbol{e}_{c}\right)\right\|^{2} \\
= & \left\|\boldsymbol{P}_{\boldsymbol{X}}^{1} \boldsymbol{e}_{a}\right\|^{2}\left\|\boldsymbol{P}_{\boldsymbol{X}}^{2} \boldsymbol{e}_{b}\right\|^{2}\left\|\boldsymbol{P}_{\boldsymbol{X}}^{3} \boldsymbol{e}_{c}\right\|^{2}+\left\|\boldsymbol{P}_{\boldsymbol{X}^{\perp}}^{1} \boldsymbol{e}_{a}\right\|^{2}\left\|\boldsymbol{P}_{\boldsymbol{X}}^{2} \boldsymbol{e}_{b}\right\|^{2}\left\|\boldsymbol{P}_{\boldsymbol{X}}^{3} \boldsymbol{e}_{c}\right\|^{2} \\
& +\left\|\boldsymbol{P}_{\boldsymbol{X}}^{1} \boldsymbol{e}_{a}\right\|^{2}\left\|\boldsymbol{P}_{\boldsymbol{X}^{\perp}}^{2} \boldsymbol{e}_{b}\right\|^{2}\left\|\boldsymbol{P}_{\boldsymbol{X}}^{3} \boldsymbol{e}_{c}\right\|^{2}+\left\|\boldsymbol{P}_{\boldsymbol{X}}^{1} \boldsymbol{e}_{a}\right\|^{2}\left\|\boldsymbol{P}_{\boldsymbol{X}}^{2} \boldsymbol{e}_{b}\right\|^{2}\left\|\boldsymbol{P}_{\boldsymbol{X}^{\perp}}^{3} \boldsymbol{e}_{c}\right\|^{2} .
\end{aligned}
$$

For brevity, write $r_{j}=r_{j}(\boldsymbol{X})$, and $\mu=\mu(\boldsymbol{X})$. Then

$$
\begin{aligned}
& \left\|\boldsymbol{P}_{\boldsymbol{X}}^{1} \boldsymbol{e}_{a}\right\|^{2}\left\|\boldsymbol{P}_{\boldsymbol{X}}^{2} \boldsymbol{e}_{b}\right\|^{2}\left\|\boldsymbol{P}_{\boldsymbol{X}}^{3} \boldsymbol{e}_{c}\right\|^{2}+\left\|\boldsymbol{P}_{\boldsymbol{X}^{\perp}}^{1} \boldsymbol{e}_{a}\right\|^{2}\left\|\boldsymbol{P}_{\boldsymbol{X}}^{2} \boldsymbol{e}_{b}\right\|^{2}\left\|\boldsymbol{P}_{\boldsymbol{X}}^{3} \boldsymbol{e}_{c}\right\|^{2} \leq \frac{r_{2} r_{3} \mu^{2}}{d_{2} d_{3}} \\
& \left\|\boldsymbol{P}_{\boldsymbol{X}}^{1} \boldsymbol{e}_{a}\right\|^{2}\left\|\boldsymbol{P}_{\boldsymbol{X}}^{2} \boldsymbol{e}_{b}\right\|^{2}\left\|\boldsymbol{P}_{\boldsymbol{X}}^{3} \boldsymbol{e}_{c}\right\|^{2}+\left\|\boldsymbol{P}_{\boldsymbol{X}}^{1} \boldsymbol{e}_{a}\right\|^{2}\left\|\boldsymbol{P}_{\boldsymbol{X}^{\perp}}^{2} \boldsymbol{e}_{b}\right\|^{2}\left\|\boldsymbol{P}_{\boldsymbol{X}}^{3} \boldsymbol{e}_{c}\right\|^{2} \leq \frac{r_{1} r_{3} \mu^{2}}{d_{1} d_{3}} \\
& \left\|\boldsymbol{P}_{\boldsymbol{X}}^{1} \boldsymbol{e}_{a}\right\|^{2}\left\|\boldsymbol{P}_{\boldsymbol{X}}^{2} \boldsymbol{e}_{b}\right\|^{2}\left\|\boldsymbol{P}_{\boldsymbol{X}}^{3} \boldsymbol{e}_{c}\right\|^{2}+\left\|\boldsymbol{P}_{\boldsymbol{X}}^{1} \boldsymbol{e}_{a}\right\|^{2}\left\|\boldsymbol{P}_{\boldsymbol{X}}^{2} \boldsymbol{e}_{b}\right\|^{2}\left\|\boldsymbol{P}_{\boldsymbol{X}^{\perp}}^{3} \boldsymbol{e}_{c}\right\|^{2} \leq \frac{r_{1} r_{2} \mu^{2}}{d_{1} d_{2}}
\end{aligned}
$$

As a result, for any $(a, b, c) \in\left[d_{1}\right] \times\left[d_{2}\right] \times\left[d_{3}\right]$,

$$
\left\|\mathcal{Q}_{\boldsymbol{X}}\left(\boldsymbol{e}_{a} \otimes \boldsymbol{e}_{b} \otimes \boldsymbol{e}_{c}\right)\right\|^{2} \leq \frac{\mu^{2}\left(r_{1} r_{2} d_{3}+r_{1} d_{2} r_{3}+d_{1} r_{2} r_{3}\right)}{d_{1} d_{2} d_{3}},
$$


which implies the desired statement.

Another measure of coherence for a tensor $\boldsymbol{X}$ is

$$
\alpha(\boldsymbol{X}):=\sqrt{d_{1} d_{2} d_{3} / \bar{r}(\boldsymbol{X})}\|\boldsymbol{W}\|_{\max }
$$

where $\boldsymbol{W}$ is such that $\boldsymbol{W}=\mathcal{Q}_{\boldsymbol{X}}^{0} \boldsymbol{W},\|\boldsymbol{W}\|=1$ and $\langle\boldsymbol{X}, \boldsymbol{W}\rangle=\|\boldsymbol{X}\|_{*}$ as described in Lemma 1. The quantity $\alpha(\boldsymbol{X})$ is related to $\mu(\boldsymbol{X})$ defined earlier and the spikiness

$$
\widetilde{\alpha}(\boldsymbol{X})=\sqrt{d_{1} d_{2} d_{3}}\|\boldsymbol{W}\|_{\max } /\|\boldsymbol{W}\|_{\mathrm{HS}}
$$

Lemma 3 Let $\boldsymbol{X} \in \mathbb{R}^{d_{1} \times d_{2} \times d_{3}}$ be a third order tensor. Assume without loss of generality that $r_{1}(\boldsymbol{X}) \leq r_{2}(\boldsymbol{X}) \leq r_{3}(\boldsymbol{X})$. Then,

$$
\alpha^{2}(\boldsymbol{X}) \leq \min \left\{r_{1}(\boldsymbol{X}) r_{2}(\boldsymbol{X}) r_{3}(\boldsymbol{X}) \mu^{3}(\boldsymbol{X}) / \bar{r}(\boldsymbol{X}), r_{1}(\boldsymbol{X}) r_{2}(\boldsymbol{X}) \widetilde{\alpha}^{2}(\boldsymbol{X}) / \bar{r}(\boldsymbol{X})\right\} .
$$

Moreover, if $\boldsymbol{X}$ admits a bi-orthogonal eigentensor decomposition $\sum_{i=1}^{r} \lambda_{i}\left(\boldsymbol{u}_{i} \otimes \boldsymbol{v}_{i} \otimes \boldsymbol{w}_{i}\right)$ with $\lambda_{i} \neq 0$ and $\boldsymbol{u}_{i}^{\top} \boldsymbol{u}_{j}=\boldsymbol{v}_{i}^{\top} \boldsymbol{v}_{j} \boldsymbol{w}_{i}^{\top} \boldsymbol{w}_{j}=\mathbb{I}\{i=j\}$ for $1 \leq i, j \leq r$, then $r_{1}(\boldsymbol{X})=r_{3}(\boldsymbol{X})=\bar{r}(\boldsymbol{X})=$ $r,\left\|X^{(1)}\right\|_{*}=\|\boldsymbol{X}\|_{*}$, and

$$
\alpha(\boldsymbol{X})=\widetilde{\alpha}(\boldsymbol{X}) \geq 1
$$

Proof of Lemma 3. Due to the conditions $\boldsymbol{W}=\mathcal{Q}_{\boldsymbol{X}}^{0} \boldsymbol{W}$ and $\|\boldsymbol{W}\|=1$,

$$
\begin{aligned}
\|\boldsymbol{W}\|_{\max }^{2} & =\max _{a, b, c}\left|\left\langle\boldsymbol{W},\left(\boldsymbol{P}_{\boldsymbol{X}}^{1} \boldsymbol{e}_{a}\right) \otimes\left(\boldsymbol{P}_{\boldsymbol{X}}^{2} \boldsymbol{e}_{b}\right) \otimes\left(\boldsymbol{P}_{\boldsymbol{X}}^{3} \boldsymbol{e}_{c}\right)\right\rangle\right|^{2} \\
& \leq \max _{a, b, c}\left\|\boldsymbol{P}_{\boldsymbol{X}}^{1} \boldsymbol{e}_{a}\right\|^{2}\left\|\boldsymbol{P}_{\boldsymbol{X}}^{2} \boldsymbol{e}_{b}\right\|^{2}\left\|\boldsymbol{P}_{\boldsymbol{X}}^{3} \boldsymbol{e}_{c}\right\|^{2} \\
& \leq r_{1}(\boldsymbol{X}) r_{2}(\boldsymbol{X}) r_{3}(\boldsymbol{X}) \mu^{3}(\boldsymbol{X}) /\left(d_{1} d_{2} d_{3}\right)
\end{aligned}
$$

which yields the upper bound for $\alpha(\boldsymbol{X})$ in terms of $\mu(\boldsymbol{X})$.

Because $\boldsymbol{W}$ is in the range of $\mathcal{Q}_{\boldsymbol{X}}^{0}, \mathcal{L}_{j}(\boldsymbol{W}) \subseteq \mathcal{L}_{j}(\boldsymbol{X})$. Therefore, $r_{1}(\boldsymbol{W}) \leq r_{1}(\boldsymbol{X})$. Recall that $W^{(1)}$ is a $d_{1} \times\left(d_{2} d_{3}\right)$ matrix whose columns are the mode- 1 fibers of $\boldsymbol{W}$. Applying singular value decomposition to $W^{(1)}$ suggests that there are orthornomal vectors $\left\{\boldsymbol{u}_{1}, \ldots, \boldsymbol{u}_{r_{1}}\right\}$ in $\mathbb{R}^{d_{1}}$ and matrices $M_{1}, \ldots, M_{r_{1}} \in \mathbb{R}^{d_{2} \times d_{3}}$ such that $\left\langle M_{j}, M_{k}\right\rangle=0$ if $j \neq k$, and

$$
\boldsymbol{W}=\sum_{k=1}^{r_{1}(\boldsymbol{X})} \boldsymbol{u}_{k} \otimes M_{k}
$$

It is clear that $\left\|M_{k}\right\| \leq\|\boldsymbol{W}\|=1$, and $\operatorname{rank}\left(M_{k}\right) \leq r_{2}(\boldsymbol{X})$. Therefore,

$$
\|\boldsymbol{W}\|_{\mathrm{HS}}^{2} \leq \sum_{k=1}^{r_{1}(\boldsymbol{X})}\left\|M_{k}\right\|_{\mathrm{HS}}^{2} \leq r_{1}(\boldsymbol{X}) r_{2}(\boldsymbol{X}) .
$$


This gives the upper bound for $\alpha(\boldsymbol{X})$ in terms of $\widetilde{\alpha}(\boldsymbol{X})$.

It remains to consider the case of $\boldsymbol{X}=\sum_{i=1}^{r} \lambda_{i}\left(\boldsymbol{u}_{i} \otimes \boldsymbol{v}_{i} \otimes \boldsymbol{w}_{i}\right)$. Obviously, by triangular inequality,

$$
\|\boldsymbol{X}\|_{*} \leq \sum_{i=1}^{r}\left\|\boldsymbol{u}_{i} \otimes \boldsymbol{v}_{i} \otimes \boldsymbol{w}_{i}\right\|_{*}=\sum_{i=1}^{r}\left\|\boldsymbol{w}_{i}\right\|
$$

On the other hand, let

$$
\boldsymbol{W}=\sum_{i=1}^{r} \boldsymbol{u}_{i} \otimes \boldsymbol{v}_{i} \otimes\left(\boldsymbol{w}_{i} /\left\|\boldsymbol{w}_{i}\right\|\right)
$$

Because

$$
\|\boldsymbol{W}\| \leq \max _{\boldsymbol{a}, \boldsymbol{b}, \boldsymbol{c}:\|\boldsymbol{a}\|,\|\boldsymbol{b}\|,\|\boldsymbol{c}\| \leq 1} \sum_{i=1}^{n}\left|\left(\boldsymbol{a}^{\top} \boldsymbol{u}_{i}\right) \operatorname{trace}\left(\boldsymbol{c} \boldsymbol{b}^{\top} \boldsymbol{v}_{i} \boldsymbol{w}_{i}^{\top} /\left\|\boldsymbol{w}_{i}\right\|\right)\right| \leq\|\boldsymbol{a}\|\left\|\boldsymbol{b} \boldsymbol{c}^{\top}\right\|_{\mathrm{HS}} \leq 1,
$$

we find

$$
\|\boldsymbol{X}\|_{*} \geq\langle\boldsymbol{W}, \boldsymbol{X}\rangle=\sum_{i=1}^{r}\left\|\boldsymbol{w}_{i}\right\|,
$$

which implies that $\boldsymbol{W}$ is dual to $\boldsymbol{X}$ and

$$
\|\boldsymbol{X}\|_{*}=\sum_{i=1}^{r}\left\|\boldsymbol{w}_{i}\right\|
$$

where the rightmost hand side also equals to $\left\|X^{(1)}\right\|_{*}$ and $\left\|X^{(2)}\right\|_{*}$. The last statement now follows from the fact that $\|\boldsymbol{W}\|_{\mathrm{HS}}^{2}=r$.

As in the matrix case, exact recovery with observations on a small fraction of the entries is only possible for tensors with low coherence. In particular, we consider in this article the recovery of a tensor $\boldsymbol{T}$ obeying $\mu(\boldsymbol{T}) \leq \mu_{0}$ and $\alpha(\boldsymbol{T}) \leq \alpha_{0}$ for some $\mu_{0}, \alpha_{0} \geq 1$.

\section{Exact Tensor Recovery}

We are now in position to study the nuclear norm minimization for tensor completion. Let $\widehat{\boldsymbol{T}}$ be the solution to

$$
\min _{\boldsymbol{X} \in \mathbb{R}^{d_{1} \times d_{2} \times d_{3}}}\|\boldsymbol{X}\|_{*} \quad \text { subject to } \mathcal{P}_{\Omega} \boldsymbol{X}=\mathcal{P}_{\Omega} \boldsymbol{T}
$$

where $\mathcal{P}_{\Omega}: \mathbb{R}^{d_{1} \times d_{2} \times d_{3}} \mapsto \mathbb{R}^{d_{1} \times d_{2} \times d_{3}}$ such that

$$
\left(\mathcal{P}_{\Omega} \boldsymbol{X}\right)(i, j, k)=\left\{\begin{array}{ll}
\boldsymbol{X}(i, j, k) & \text { if }(i, j, k) \in \Omega \\
0 & \text { otherwise }
\end{array} .\right.
$$


Assume that $\Omega$ is a uniformly sampled subset of $\left[d_{1}\right] \times\left[d_{2}\right] \times\left[d_{3}\right]$. The goal is to determine what the necessary sample size is for successful reconstruction of $\boldsymbol{T}$ using $\widehat{\boldsymbol{T}}$ with high probability. In particular, we show that that with high probability, exact recovery can be achieved with nuclear norm minimization (3) if

$$
|\Omega| \geq\left(\alpha_{0} \sqrt{r d_{1} d_{2} d_{3}}+\mu_{0}^{2} r^{2} d\right) \operatorname{polylog}(d)
$$

where $d=d_{1}+d_{2}+d_{3}$. More specifically, we have

Theorem 1 Assume that $\mu(\boldsymbol{T}) \leq \mu_{0}, \alpha(\boldsymbol{T}) \leq \alpha_{0}$, and $\bar{r}(\boldsymbol{T})=r$. Let $\Omega$ be a uniformly sampled subset of $\left[d_{1}\right] \times\left[d_{2}\right] \times\left[d_{3}\right]$ and $\widehat{\boldsymbol{T}}$ be the solution to (3). For $\beta>0$, define

$$
q_{1}^{*}=(\beta+\log d)^{2} \alpha_{0}^{2} r \log d, \quad q_{2}^{*}=(1+\beta)(\log d) \mu_{0}^{2} r^{2} .
$$

Let $n=|\Omega|$. Suppose that for a sufficiently large numerical constant $c_{0}$,

$$
n \geq c_{0} \delta_{2}^{-1}\left[\sqrt{q_{1}^{*}(1+\beta) \delta_{1}^{-1} d_{1} d_{2} d_{3}}+q_{1}^{*} d^{1+\delta_{1}}+q_{2}^{*} d^{1+\delta_{2}}\right]
$$

with certain $\left\{\delta_{1}, \delta_{2}\right\} \in[1 / \log d, 1 / 2]$ and $\beta>0$. Then,

$$
\mathbb{P}\{\widehat{\boldsymbol{T}} \neq \boldsymbol{T}\} \leq d^{-\beta}
$$

In particular, for $\delta_{1}=\delta_{2}=(\log d)^{-1}$, (4) can be written as

$$
n \geq C_{\mu_{0}, \alpha_{0}, \beta}\left[(\log d)^{3} \sqrt{r d_{1} d_{2} d_{3}}+\left\{r(\log d)^{3}+r^{2}(\log d)\right\} d\right]
$$

with a constant $C_{\mu_{0}, \alpha_{0}, \beta}$ depending on $\left\{\mu_{0}, \alpha_{0}, \beta\right\}$ only.

For $d_{1} \asymp d_{2} \asymp d_{3}$ and fixed $\left\{\alpha_{0}, \mu_{0}, \delta_{1}, \delta_{2}, \beta\right\}$, the sample size requirement (4) becomes

$$
n \asymp \sqrt{r}(d \log d)^{3 / 2},
$$

provided $\max \left\{r(\log d)^{3} d^{2 \delta_{1}}, r^{3} d^{2 \delta_{2}} /(\log d)\right\}=O(d)$.

The high level idea of our strategy is similar to the matrix case - exact recovery of $\boldsymbol{T}$ is implied by the existence of a dual certificate $\boldsymbol{G}$ supported on $\Omega$, that is $\mathcal{P}_{\Omega} \boldsymbol{G}=\boldsymbol{G}$, such that $\mathcal{Q}_{\boldsymbol{T}} \boldsymbol{G}=\boldsymbol{W}$ and $\left\|\mathcal{Q}_{\boldsymbol{T}^{\perp}} \boldsymbol{G}\right\|<1 / 2$.

\subsection{Recovery with a Dual Certificate}

Write $\widehat{\boldsymbol{T}}=\boldsymbol{T}+\Delta$. Then, $\mathcal{P}_{\Omega} \Delta=0$ and

$$
\|\boldsymbol{T}+\Delta\|_{*} \leq\|\boldsymbol{T}\|_{*}
$$


Recall that, by Lemma 1, there exists a $\boldsymbol{W}$ obeying $\boldsymbol{W}=\mathcal{Q}_{\boldsymbol{T}}^{0} \boldsymbol{W}$ and $\|\boldsymbol{W}\|=1$ such that

$$
\|\boldsymbol{T}+\Delta\|_{*} \geq\|\boldsymbol{T}\|_{*}+\left\langle\boldsymbol{W}+\mathcal{Q}_{\boldsymbol{T}^{\perp}} \boldsymbol{W}^{\perp}, \Delta\right\rangle
$$

for any $\boldsymbol{W}^{\perp}$ obeying $\left\|\boldsymbol{W}^{\perp}\right\| \leq 1 / 2$. Assume that a tensor $\boldsymbol{G}$ supported on $\Omega$, that is $\mathcal{P}_{\Omega} \boldsymbol{G}=\boldsymbol{G}$, such that $\mathcal{Q}_{\boldsymbol{T}} \boldsymbol{G}=\boldsymbol{W}$, and $\left\|\mathcal{Q}_{\boldsymbol{T}^{\perp}} \boldsymbol{G}\right\|<1 / 2$. When $\left\|\mathcal{Q}_{\boldsymbol{T}^{\perp}} \Delta\right\|_{*}>0$,

$$
\begin{aligned}
\left\langle\boldsymbol{W}+\mathcal{Q}_{T^{\perp}} \boldsymbol{W}^{\perp}, \Delta\right\rangle & =\left\langle\boldsymbol{W}+\mathcal{Q}_{T^{\perp}} \boldsymbol{W}^{\perp}-\boldsymbol{G}, \Delta\right\rangle \\
& =\left\langle\boldsymbol{W}-\mathcal{Q}_{T} \boldsymbol{G}, \Delta\right\rangle+\left\langle\boldsymbol{W}^{\perp}, \mathcal{Q}_{\boldsymbol{T}^{\perp}} \Delta\right\rangle-\left\langle\mathcal{Q}_{\boldsymbol{T}^{\perp}} \boldsymbol{G}, \mathcal{Q}_{\boldsymbol{T}^{\perp}} \Delta\right\rangle \\
& >\left\langle\boldsymbol{W}^{\perp}, \mathcal{Q}_{\boldsymbol{T}^{\perp}} \Delta\right\rangle-\frac{1}{2}\left\|\mathcal{Q}_{\boldsymbol{T}^{\perp}} \Delta\right\|_{*}
\end{aligned}
$$

Take $\boldsymbol{W}^{\perp}=\boldsymbol{U} / 2$ where

$$
\boldsymbol{U}=\underset{\boldsymbol{X}:\|\boldsymbol{X}\| \leq 1}{\arg \max }\left\langle\boldsymbol{X}, \mathcal{Q}_{T^{\perp}} \Delta\right\rangle .
$$

We find that $\left\|\mathcal{Q}_{T^{\perp}} \Delta\right\|_{*}>0$ implies

$$
\|\boldsymbol{T}+\Delta\|_{*}-\|\boldsymbol{T}\|_{*} \geq\left\langle\boldsymbol{W}+\mathcal{Q}_{T^{\perp}} \boldsymbol{W}^{\perp}, \Delta\right\rangle>0,
$$

which contradicts with fact that $\widehat{\boldsymbol{T}}$ minimizes the nuclear norm. Thus, $\mathcal{Q}_{T^{\perp}} \Delta=0$, which then implies $\mathcal{Q}_{T} \mathcal{Q}_{\Omega} \mathcal{Q}_{T} \Delta=\mathcal{Q}_{T} \mathcal{Q}_{\Omega} \Delta=0$. When $\mathcal{Q}_{T} \mathcal{Q}_{\Omega} \mathcal{Q}_{T}$ is invertible in the range of $\mathcal{Q}_{T}$, we also have $\mathcal{Q}_{T} \Delta=0$ and $\widehat{\boldsymbol{T}}=\boldsymbol{T}$.

With this in mind, it then suffices to seek such a dual certificate. In fact, it turns out that finding an "approximate" dual certificate is actually enough for our purposes.

Lemma 4 Assume that

$$
\inf \left\{\left\|\mathcal{P}_{\Omega} \mathcal{Q}_{\boldsymbol{T}} \boldsymbol{X}\right\|_{\mathrm{HS}}:\left\|\mathcal{Q}_{\boldsymbol{T}} \boldsymbol{X}\right\|_{\mathrm{HS}}=1\right\} \geq \sqrt{\frac{n}{2 d_{1} d_{2} d_{3}}} .
$$

If there exists a tensor $\boldsymbol{G}$ supported on $\Omega$ such that

$$
\left\|\mathcal{Q}_{\boldsymbol{T}} \boldsymbol{G}-\boldsymbol{W}\right\|_{\mathrm{HS}}<\frac{1}{4} \sqrt{\frac{n}{2 d_{1} d_{2} d_{3}}} \quad \text { and } \quad \max _{\left\|\mathcal{Q}_{\boldsymbol{T}^{\perp}} \boldsymbol{X}\right\|_{*}=1}\left\langle\boldsymbol{G}, \mathcal{Q}_{\boldsymbol{T}^{\perp}} \boldsymbol{X}\right\rangle \leq 1 / 4,
$$

then $\widehat{\boldsymbol{T}}=\boldsymbol{T}$.

Proof of Lemma 4. Write $\widehat{\boldsymbol{T}}=\boldsymbol{T}+\boldsymbol{\Delta}$, then $\mathcal{P}_{\Omega} \boldsymbol{\Delta}=0$ and

$$
\|\boldsymbol{T}+\Delta\|_{*} \leq\|\boldsymbol{T}\|_{*} .
$$

Recall that, by Lemma 1, there exists a $\boldsymbol{W}$ obeying $\boldsymbol{W}=\mathcal{Q}_{\boldsymbol{T}}^{0} \boldsymbol{W}$ and $\|\boldsymbol{W}\|=1$ such that for any $\boldsymbol{W}^{\perp}$ obeying $\left\|\boldsymbol{W}^{\perp}\right\| \leq 1 / 2$,

$$
\|\boldsymbol{T}+\boldsymbol{\Delta}\|_{*} \geq\|\boldsymbol{T}\|_{*}+\left\langle\boldsymbol{W}+\mathcal{Q}_{\boldsymbol{T}^{\perp}} \boldsymbol{W}^{\perp}, \boldsymbol{\Delta}\right\rangle .
$$


Since $\langle\boldsymbol{G}, \boldsymbol{\Delta}\rangle=\left\langle\mathcal{P}_{\Omega} \boldsymbol{G}, \boldsymbol{\Delta}\right\rangle=\left\langle\boldsymbol{G}, \mathcal{P}_{\Omega} \boldsymbol{\Delta}\right\rangle=0$ and $\mathcal{Q}_{\boldsymbol{T}} \boldsymbol{W}=\boldsymbol{W}$,

$$
\begin{aligned}
0 & \geq\left\langle\boldsymbol{W}+\mathcal{Q}_{T^{\perp}} \boldsymbol{W}^{\perp}, \boldsymbol{\Delta}\right\rangle \\
& =\left\langle\boldsymbol{W}+\mathcal{Q}_{T^{\perp}} \boldsymbol{W}^{\perp}-\boldsymbol{G}, \boldsymbol{\Delta}\right\rangle \\
& =\left\langle\mathcal{Q}_{\boldsymbol{T}} \boldsymbol{W}-\mathcal{Q}_{\boldsymbol{T}} \boldsymbol{G}, \boldsymbol{\Delta}\right\rangle+\left\langle\boldsymbol{W}^{\perp}, \mathcal{Q}_{T^{\perp}} \boldsymbol{\Delta}\right\rangle-\left\langle\boldsymbol{G}, \mathcal{Q}_{T^{\perp}} \boldsymbol{\Delta}\right\rangle \\
& \geq-\left\|\boldsymbol{W}-\mathcal{Q}_{\boldsymbol{T}} \boldsymbol{G}\right\|_{\mathrm{HS}}\left\|\mathcal{Q}_{\boldsymbol{T}} \boldsymbol{\Delta}\right\|_{\mathrm{HS}}+\left\langle\boldsymbol{W}^{\perp}, \mathcal{Q}_{T^{\perp}} \boldsymbol{\Delta}\right\rangle-\frac{1}{4}\left\|\mathcal{Q}_{\boldsymbol{T}^{\perp}} \boldsymbol{\Delta}\right\|_{*} .
\end{aligned}
$$

In particular, taking $\boldsymbol{W}^{\perp}$ satisfying $\|\boldsymbol{W}\|=1 / 2$ and $\left\langle\boldsymbol{W}^{\perp}, \mathcal{Q}_{\boldsymbol{T}^{\perp}} \boldsymbol{\Delta}\right\rangle=\left\|\mathcal{Q}_{\boldsymbol{T}^{\perp}} \boldsymbol{\Delta}\right\|_{*} / 2$, we find

$$
\frac{1}{4}\left\|\mathcal{Q}_{\boldsymbol{T}^{\perp}} \boldsymbol{\Delta}\right\|_{*} \leq\left\|\boldsymbol{W}-\mathcal{Q}_{\boldsymbol{T}} \boldsymbol{G}\right\|_{\mathrm{HS}}\left\|\mathcal{Q}_{\boldsymbol{T}} \boldsymbol{\Delta}\right\|_{\mathrm{HS}}
$$

Recall that $\mathcal{P}_{\Omega} \Delta=\mathcal{P}_{\Omega} \mathcal{Q}_{T^{\perp}} \Delta+\mathcal{P}_{\Omega} \mathcal{Q}_{T} \Delta=0$. Thus, in view of the condition on $\mathcal{P}_{\Omega}$,

$$
\frac{\left\|\mathcal{Q}_{T} \boldsymbol{\Delta}\right\|_{\mathrm{HS}}}{\sqrt{2 d_{1} d_{2} d_{3} / n}} \leq\left\|\mathcal{P}_{\Omega} \mathcal{Q}_{T} \boldsymbol{\Delta}\right\|_{\mathrm{HS}}=\left\|\mathcal{P}_{\Omega} \mathcal{Q}_{T^{\perp}} \boldsymbol{\Delta}\right\|_{\mathrm{HS}} \leq\left\|\mathcal{Q}_{T^{\perp}} \boldsymbol{\Delta}\right\|_{\mathrm{HS}} \leq\left\|\mathcal{Q}_{T^{\perp}} \boldsymbol{\Delta}\right\|_{*} .
$$

Consequently,

Since

$$
\frac{1}{4}\left\|\mathcal{Q}_{T^{\perp}} \boldsymbol{\Delta}\right\|_{*} \leq \sqrt{2 d_{1} d_{2} d_{3} / n}\left\|\boldsymbol{W}-\mathcal{Q}_{T} \boldsymbol{G}\right\|_{\mathrm{HS}}\left\|\mathcal{Q}_{T^{\perp}} \boldsymbol{\Delta}\right\|_{*} .
$$

$$
\sqrt{2 d_{1} d_{2} d_{3} / n}\left\|\boldsymbol{W}-\mathcal{Q}_{T} \boldsymbol{G}\right\|_{\mathrm{HS}}<1 / 4
$$

we have $\left\|\mathcal{Q}_{\boldsymbol{T}^{\perp}} \boldsymbol{\Delta}\right\|_{*}=0$. Together with (8) , we conclude that $\boldsymbol{\Delta}=0$, or equivalently $\widehat{\boldsymbol{T}}=\boldsymbol{T}$.

Equation (66) indicates the invertibility of $\mathcal{P}_{\Omega}$ when restricted to the range of $\mathcal{Q}_{\boldsymbol{T}}$. We argue first that this is true for "incoherent" tensors. To this end, we prove that

$$
\left\|\mathcal{Q}_{T}\left(\left(d_{1} d_{2} d_{3} / n\right) \mathcal{P}_{\Omega}-\mathcal{I}\right) \mathcal{Q}_{T}\right\| \leq 1 / 2
$$

with high probability. This implies that as an operator in the range of $\mathcal{Q}_{\boldsymbol{T}}$, the spectral norm of $\left(d_{1} d_{2} d_{3} / n\right) \mathcal{Q}_{\boldsymbol{T}} \mathcal{P}_{\Omega} \mathcal{Q}_{\boldsymbol{T}}$ is contained in [1/2,3/2]. Consequently, (6) holds because for any $\boldsymbol{X} \in \mathbb{R}^{d_{1} \times d_{2} \times d_{3}}$,

$$
\left(d_{1} d_{2} d_{3} / n\right)\left\|\mathcal{P}_{\Omega} \mathcal{Q}_{\boldsymbol{T}} \boldsymbol{X}\right\|_{\mathrm{HS}}^{2}=\left\langle\mathcal{Q}_{\boldsymbol{T}} \boldsymbol{X},\left(d_{1} d_{2} d_{3} / n\right) \mathcal{Q}_{\boldsymbol{T}} \mathcal{P}_{\Omega} \mathcal{Q}_{\boldsymbol{T}} \boldsymbol{X}\right\rangle \geq \frac{1}{2}\left\|\mathcal{Q}_{\boldsymbol{T}} \boldsymbol{X}\right\|_{\mathrm{HS}}^{2} .
$$

Recall that $d=d_{1}+d_{2}+d_{3}$. We have

Lemma 5 Assume $\mu(\boldsymbol{T}) \leq \mu_{0}, \bar{r}(\boldsymbol{T})=r$, and $\Omega$ is uniformly sampled from $\left[d_{1}\right] \times\left[d_{2}\right] \times\left[d_{3}\right]$ without replacement. Then, for any $\tau>0$,

$$
\mathbb{P}\left\{\left\|\mathcal{Q}_{T}\left(\left(d_{1} d_{2} d_{3} / n\right) \mathcal{P}_{\Omega}-\mathcal{I}\right) \mathcal{Q}_{T}\right\| \geq \tau\right\} \leq 2 r^{2} d \exp \left(-\frac{\tau^{2} / 2}{1+2 \tau / 3}\left(\frac{n}{\mu_{0}^{2} r^{2} d}\right)\right) .
$$

In particular, taking $\tau=1 / 2$ in Lemma 5 yields

$$
\mathbb{P}\{\text { (6) holds }\} \geq 1-2 r^{2} d \exp \left(-\frac{3}{32}\left(\frac{n}{\mu_{0}^{2} r^{2} d}\right)\right) .
$$




\subsection{Constructing a Dual Certificate}

We now show that the "approximate" dual certificate as required by Lemma 4 can indeed be constructed when $\Omega$ is a uniformly sampled subset of $\left[d_{1}\right] \times\left[d_{2}\right] \times\left[d_{3}\right]$. We use a strategy similar to the "golfing scheme" for the matrix case (see, e.g., Gross, 2011).

We begin by constructing an iid uniformly distributed sequence in $\left[d_{1}\right] \times\left[d_{2}\right] \times\left[d_{3}\right]$, $\left\{\left(a_{i}, b_{i}, c_{i}\right): 1 \leq i \leq n\right\}$. This can be done by sampling with replacement from $\Omega$ :

\section{Creating IID Samples from $\Omega$}

- Initialize $S_{0}=\emptyset$.

- For each $i=1,2, \ldots, n$,

- with probability $\left|S_{i-1}\right| / d_{1} d_{2} d_{3}$, sample $\left(a_{i}, b_{i}, c_{i}\right)$ uniformly from $S_{i-1}$; and with probability $1-\left|S_{i-1}\right| / d_{1} d_{2} d_{3}$, sample $\left(a_{i}, b_{i}, c_{i}\right)$ uniformly from $\Omega \backslash S_{i-1}$.

- Update $S_{i}=S_{i-1} \cup\left\{\left(a_{i}, b_{i}, c_{i}\right)\right\}$

Because $\mathbb{P}\left\{\left(a_{i}, b_{i}, c_{i}\right) \in S_{i-1} \mid S_{i-1}\right\}$ matches that of the iid case and $\left(a_{i}, b_{i}, c_{i}\right)$ is uniform in $\left[d_{1}\right] \times\left[d_{2}\right] \times\left[d_{3}\right] \backslash S_{i-1}$ conditionally on $S_{i-1}$ and $\left(a_{i}, b_{i}, c_{i}\right) \notin S_{i-1}$, the points $\left(a_{i}, b_{i}, c_{i}\right)$ are iid uniform in $\left[d_{1}\right] \times\left[d_{2}\right] \times\left[d_{3}\right]$.

We now divide the sequence $\left\{\left(a_{i}, b_{i}, c_{i}\right): 1 \leq i \leq n\right\}$ into $n_{2}$ subsequences of length $n_{1}$ :

$$
\Omega_{k}=\left\{\left(a_{i}, b_{i}, c_{i}\right):(k-1) n_{1}<i \leq k n_{1}\right\},
$$

for $k=1,2, \ldots, n_{2}$, where $n_{1} n_{2} \leq n$. Recall that $\boldsymbol{W}$ is such that $\boldsymbol{W}=\mathcal{Q}_{\boldsymbol{T}}^{0} \boldsymbol{W},\|\boldsymbol{W}\|=1$, and $\|\boldsymbol{T}\|_{*}=\langle\boldsymbol{T}, \boldsymbol{W}\rangle$. Let

$$
\mathcal{R}_{k}=\mathcal{I}-\frac{1}{n_{1}} \sum_{i=(k-1) n_{1}+1}^{k n_{1}}\left(d_{1} d_{2} d_{3}\right) \mathcal{P}_{\left(a_{i}, b_{i}, c_{i}\right)}
$$

with $\mathcal{I}$ being the identity operator on tensors and define

$$
\boldsymbol{G}_{k}=\sum_{\ell=1}^{k}\left(\mathcal{I}-\mathcal{R}_{\ell}\right) \mathcal{Q}_{\boldsymbol{T}} \mathcal{R}_{\ell-1} \mathcal{Q}_{\boldsymbol{T}} \cdots \mathcal{Q}_{\boldsymbol{T}} \mathcal{R}_{1} \mathcal{Q}_{\boldsymbol{T}} \boldsymbol{W}, \quad \boldsymbol{G}=\boldsymbol{G}_{n_{2}}
$$

Since $\left(a_{i}, b_{i}, c_{i}\right) \in \Omega, \mathcal{P}_{\Omega}\left(\mathcal{I}-\mathcal{R}_{k}\right)=\mathcal{I}-\mathcal{R}_{k}$, so that $\mathcal{P}_{\Omega} \boldsymbol{G}=\boldsymbol{G}$. It follows from the definition of $\boldsymbol{G}_{k}$ that

$$
\mathcal{Q}_{\boldsymbol{T}} \boldsymbol{G}_{k}=\sum_{\ell=1}^{k}\left(\mathcal{Q}_{\boldsymbol{T}}-\mathcal{Q}_{\boldsymbol{T}} \mathcal{R}_{\ell} \mathcal{Q}_{\boldsymbol{T}}\right)\left(\mathcal{Q}_{\boldsymbol{T}} \mathcal{R}_{\ell-1} \mathcal{Q}_{\boldsymbol{T}}\right) \cdots\left(\mathcal{Q}_{\boldsymbol{T}} \mathcal{R}_{1} \mathcal{Q}_{\boldsymbol{T}} \boldsymbol{W}\right)
$$




$$
=\boldsymbol{W}-\left(\mathcal{Q}_{\boldsymbol{T}} \mathcal{R}_{k} \mathcal{Q}_{\boldsymbol{T}}\right) \cdots\left(\mathcal{Q}_{\boldsymbol{T}} \mathcal{R}_{1} \mathcal{Q}_{\boldsymbol{T}}\right) \boldsymbol{W}
$$

and

$$
\left\langle\boldsymbol{G}_{k}, \mathcal{Q}_{\boldsymbol{T}^{\perp}} \boldsymbol{X}\right\rangle=\left\langle\sum_{\ell=1}^{k} \mathcal{R}_{\ell}\left(\mathcal{Q}_{\boldsymbol{T}} \mathcal{R}_{\ell-1} \mathcal{Q}_{\boldsymbol{T}}\right) \cdots\left(\mathcal{Q}_{\boldsymbol{T}} \mathcal{R}_{1} \mathcal{Q}_{\boldsymbol{T}}\right) \boldsymbol{W}, \mathcal{Q}_{\boldsymbol{T}^{\perp}} \boldsymbol{X}\right\rangle .
$$

Thus, condition (7) holds if

$$
\left\|\left(\mathcal{Q}_{\boldsymbol{T}} \mathcal{R}_{n_{2}} \mathcal{Q}_{\boldsymbol{T}}\right) \cdots\left(\mathcal{Q}_{\boldsymbol{T}} \mathcal{R}_{1} \mathcal{Q}_{\boldsymbol{T}}\right) \boldsymbol{W}\right\|_{\mathrm{HS}}<\frac{1}{4} \sqrt{\frac{n}{2 d_{1} d_{2} d_{3}}}
$$

and

$$
\left\|\sum_{\ell=1}^{n_{2}} \mathcal{R}_{\ell}\left(\mathcal{Q}_{\boldsymbol{T}} \mathcal{R}_{\ell-1} \mathcal{Q}_{\boldsymbol{T}}\right) \cdots\left(\mathcal{Q}_{\boldsymbol{T}} \mathcal{R}_{1} \mathcal{Q}_{\boldsymbol{T}}\right) \boldsymbol{W}\right\|<1 / 4
$$

\subsection{Verifying Conditions for Dual Certificate}

We now prove that (9) and (10) hold with high probability for the approximate dual certificate constructed above. For this purpose, we need large deviation bounds for the average of certain iid tensors under the spectral and maximum norms.

Lemma 6 Let $\left\{\left(a_{i}, b_{i}, c_{i}\right)\right\}$ be an independently and uniformly sampled sequence from $\left[d_{1}\right] \times$ $\left[d_{2}\right] \times\left[d_{3}\right]$. Assume that $\mu(\boldsymbol{T}) \leq \mu_{0}$ and $\bar{r}(\boldsymbol{T})=r$. Then, for any fixed $k=1,2, \ldots, n_{2}$, and for all $\tau>0$,

$$
\mathbb{P}\left\{\left\|\mathcal{Q}_{\boldsymbol{T}} \mathcal{R}_{k} \mathcal{Q}_{\boldsymbol{T}}\right\| \geq \tau\right\} \leq 2 r^{2} d \exp \left(-\frac{\tau^{2} / 2}{1+2 \tau / 3}\left(\frac{n_{1}}{\mu_{0}^{2} r^{2} d}\right)\right),
$$

and

$$
\max _{\|\boldsymbol{X}\|_{\max }=1} \mathbb{P}\left\{\left\|\mathcal{Q}_{\boldsymbol{T}} \mathcal{R}_{k} \mathcal{Q}_{\boldsymbol{T}} \boldsymbol{X}\right\|_{\max } \geq \tau\right\} \leq 2 d_{1} d_{2} d_{3} \exp \left(-\frac{\tau^{2} / 2}{1+2 \tau / 3}\left(\frac{n_{1}}{\mu_{0}^{2} r^{2} d}\right)\right) .
$$

Because

$$
\left(d_{1} d_{2} d_{3}\right)^{-1 / 2}\|\boldsymbol{W}\|_{\mathrm{HS}} \leq\|\boldsymbol{W}\|_{\max } \leq\|\boldsymbol{W}\| \leq 1,
$$

Equation (9) holds if $\max _{1 \leq \ell \leq n_{2}}\left\|\mathcal{Q}_{\boldsymbol{T}} \mathcal{R}_{\ell} \mathcal{Q}_{\boldsymbol{T}}\right\| \leq \tau$ and

$$
n_{2} \geq-\frac{1}{\log \tau} \log \left(\sqrt{32} d_{1} d_{2} d_{3} n^{-1 / 2}\right) .
$$

Thus, an application of (11) now gives the following bound:

$$
\begin{aligned}
\mathbb{P}\{(9) \text { holds }\} & \geq 1-\mathbb{P}\left\{\left\|\left(\mathcal{Q}_{\boldsymbol{T}} \mathcal{R}_{n_{2}} \mathcal{Q}_{\boldsymbol{T}}\right) \cdots\left(\mathcal{Q}_{\boldsymbol{T}} \mathcal{R}_{1} \mathcal{Q}_{\boldsymbol{T}}\right)\right\| \geq \tau^{n_{2}}\right\} \\
& \leq 1-\mathbb{P}\left\{\max _{1 \leq \ell \leq n_{2}}\left\|\mathcal{Q}_{\boldsymbol{T}} \mathcal{R}_{\ell} \mathcal{Q}_{\boldsymbol{T}}\right\| \geq \tau\right\} \\
& \leq 1-2 n_{2} r^{2} d \exp \left(-\frac{\tau^{2} / 2}{1+2 \tau / 3}\left(\frac{n_{1}}{\mu_{0}^{2} r^{2} d}\right)\right) .
\end{aligned}
$$


Now consider Equation (10). Let $\boldsymbol{W}_{\ell}=\mathcal{Q}_{\boldsymbol{T}} \mathcal{R}_{\ell} \mathcal{Q}_{\boldsymbol{T}} \boldsymbol{W}_{\ell-1}$ for $\ell \geq 1$ with $\boldsymbol{W}_{0}=\boldsymbol{W}$. Observe that (10) does not hold with at most probability

$$
\begin{aligned}
& \mathbb{P}\left\{\left\|\sum_{\ell=1}^{n_{2}} \mathcal{R}_{\ell} \boldsymbol{W}_{\ell-1}\right\| \geq 1 / 4\right\} \\
\leq & \mathbb{P}\left\{\left\|\mathcal{R}_{1} \boldsymbol{W}_{0}\right\| \geq 1 / 8\right\}+\mathbb{P}\left\{\left\|\boldsymbol{W}_{1}\right\|_{\max } \geq\|\boldsymbol{W}\|_{\max } / 2\right\} \\
& +\mathbb{P}\left\{\left\|\sum_{\ell=2}^{n_{2}} \mathcal{R}_{\ell} \boldsymbol{W}_{\ell-1}\right\| \geq 1 / 8,\left\|\boldsymbol{W}_{1}\right\|_{\max }<\|\boldsymbol{W}\|_{\max } / 2\right\} \\
\leq & \mathbb{P}\left\{\left\|\mathcal{R}_{1} \boldsymbol{W}_{0}\right\| \geq 1 / 8\right\}+\mathbb{P}\left\{\left\|\boldsymbol{W}_{1}\right\|_{\max } \geq\|\boldsymbol{W}\|_{\max } / 2\right\} \\
& +\mathbb{P}\left\{\left\|\mathcal{R}_{2} \boldsymbol{W}_{1}\right\| \geq 1 / 16,\left\|\boldsymbol{W}_{1}\right\|_{\max }<\|\boldsymbol{W}\|_{\max } / 2\right\} \\
& +\mathbb{P}\left\{\left\|\boldsymbol{W}_{2}\right\|_{\max } \geq\|\boldsymbol{W}\|_{\max } / 4,\left\|\boldsymbol{W}_{1}\right\|_{\max }<\|\boldsymbol{W}\|_{\max } / 2\right\} \\
& +\mathbb{P}\left\{\left\|\sum_{\ell=3}^{n_{2}} \mathcal{R}_{\ell} \boldsymbol{W}_{\ell-1}\right\| \geq \frac{1}{16},\left\|\boldsymbol{W}_{2}\right\|_{\max }<\|\boldsymbol{W}\|_{\max } / 4\right\} \\
& \sum_{\ell=1}^{n_{2}-1} \mathbb{P}\left\{\left\|\mathcal{Q}_{\boldsymbol{T}} \mathcal{R}_{\ell} \mathcal{Q}_{\boldsymbol{T}} \boldsymbol{W}_{\ell-1}\right\|_{\max } \geq\left\|\boldsymbol{W}_{\max } / 2^{\ell},\right\| \boldsymbol{W}_{\ell-1}\left\|_{\max } \leq\right\| \boldsymbol{W} \|_{\max } / 2^{\ell-1}\right\} \\
& +\sum_{\ell=1}^{n_{2}} \mathbb{P}\left\{\left\|\mathcal{R}_{\ell} \boldsymbol{W}_{\ell-1}\right\| \geq 2^{-2-\ell},\left\|\boldsymbol{W}_{\ell-1}\right\|_{\max } \leq \| \boldsymbol{W}_{\max } / 2^{\ell-1}\right\} .
\end{aligned}
$$

Since $\left\{\mathcal{R}_{\ell}, \boldsymbol{W}_{\ell}\right\}$ are i.i.d., (12) with $\boldsymbol{X}=\boldsymbol{W}_{\ell-1} /\left\|\boldsymbol{W}_{\ell-1}\right\|_{\max }$ implies

$$
\begin{aligned}
& \mathbb{P}\{(\underline{10}) \text { holds }\} \\
\geq & 1-n_{2} \max _{\substack{\boldsymbol{X}: \boldsymbol{X}_{\|}=\mathcal{Q}_{\boldsymbol{T}} \boldsymbol{X} \\
\|\boldsymbol{X}\|_{\max } \leq 1}}\left(\mathbb{P}\left\{\left\|\mathcal{Q}_{\boldsymbol{T}} \mathcal{R}_{1} \mathcal{Q}_{\boldsymbol{T}} \boldsymbol{X}\right\|_{\max }>\frac{1}{2}\right\}+\mathbb{P}\left\{\left\|\mathcal{R}_{1} \boldsymbol{X}\right\|>\frac{1}{8\|\boldsymbol{W}\|_{\max }}\right\}\right) \\
\geq & 1-2 n_{2} d_{1} d_{2} d_{3} \exp \left(\frac{-(3 / 32) n_{1}}{\mu_{0}^{2} r^{2} d}\right)-n_{2} \underset{\substack{\boldsymbol{X}: \boldsymbol{X}=\mathcal{Q}_{\boldsymbol{T}} \boldsymbol{X} \\
\|\boldsymbol{X}\|_{\max } \leq\|\boldsymbol{W}\|_{\max }}}{\max } \mathbb{P}\left\{\left\|\mathcal{R}_{1} \boldsymbol{X}\right\|>\frac{1}{8}\right\} .
\end{aligned}
$$

The last term on the right hand side can be bounded using the following result.

Lemma 7 Assume that $\alpha(\boldsymbol{T}) \leq \alpha_{0}, \bar{r}(\boldsymbol{T})=r$ and $q_{1}^{*}=(\beta+\log d)^{2} \alpha_{0}^{2} r \log d$. There exists a numerical constant $c_{1}>0$ such that for any constants $\beta>0$ and $1 /(\log d) \leq \delta_{1}<1$,

$$
n_{1} \geq c_{1}\left[q_{1}^{*} d^{1+\delta_{1}}+\sqrt{q_{1}^{*}(1+\beta) \delta_{1}^{-1} d_{1} d_{2} d_{3}}\right]
$$

implies

$$
\max _{\substack{\boldsymbol{X}: \boldsymbol{X}=\mathcal{Q}_{\boldsymbol{T}} \boldsymbol{X} \\\|\boldsymbol{X}\|_{\max } \leq\|\boldsymbol{W}\|_{\max }}} \mathbb{P}\left\{\left\|\mathcal{R}_{1} \boldsymbol{X}\right\| \geq \frac{1}{8}\right\} \leq d^{-\beta-1}
$$


where $\boldsymbol{W}$ is in the range of $\mathcal{Q}_{\boldsymbol{T}}^{0}$ such that $\|\boldsymbol{W}\|=1$ and $\langle\boldsymbol{T}, \boldsymbol{W}\rangle=\|\boldsymbol{T}\|_{*}$.

\subsection{Proof of Theorem 1}

Since (7) is a consequence of (9) and (10), it follows from Lemmas 4, 5, 6 and 7 that for $\tau \in(0,1 / 2]$ and $n \geq n_{1} n_{2}$ satisfying conditions (13) and (14),

$$
\begin{aligned}
\mathbb{P}\{\widehat{\boldsymbol{T}} \neq \boldsymbol{T}\} \leq & 2 r^{2} d \exp \left(-\frac{3}{32}\left(\frac{n}{\mu_{0}^{2} r^{2} d}\right)\right)+2 n_{2} r^{2} d \exp \left(-\frac{\tau^{2} / 2}{1+2 \tau / 3}\left(\frac{n_{1}}{\mu_{0}^{2} r^{2} d}\right)\right) \\
& +2 n_{2} d_{1} d_{2} d_{3} \exp \left(\frac{-(3 / 32) n_{1}}{\mu_{0}^{2} r^{2} d}\right)+n_{2} d^{-\beta-1} .
\end{aligned}
$$

We now prove Theorem 11 by setting $\tau=d^{-\delta_{2} / 2} / 2$, so that condition (13) can be written as $n_{2} \geq c_{2} / \delta_{2}$. Assume without loss of generality $n_{2} \leq d / 2$ because large $c_{0}$ forces large $d$. For sufficiently large $c_{2}^{\prime}$, the right-hand side of the above inequality is no greater than $d^{-\beta}$ when

$$
n_{1} \geq c_{2}^{\prime}(1+\beta)(\log d) \mu_{0}^{2} r^{2} d /\left(4 \tau^{2}\right)=c_{2}^{\prime} q_{2}^{*} d^{1+\delta_{2}}
$$

holds as well as (14). Thus, (44) implies (5) for sufficiently large $c_{0}$.

\section{Concentration Inequalities for Low Rank Tensors}

We now prove Lemmas 5 and 6 , both involving tensors of low rank. We note that Lemma 5 concerns the concentration inequality for the sum of a sequence of dependent tensors whereas in Lemma 6, we are interested in a sequence of iid tensors.

\subsection{Proof of Lemma 5}

We first consider Lemma 5. Let $\left(a_{k}, b_{k}, c_{k}\right)$ be sequentially uniformly sampled from $\Omega^{*}$ without replacement, $S_{k}=\left\{\left(a_{j}, b_{j}, c_{j}\right): j \leq k\right\}$, and $m_{k}=d_{1} d_{2} d_{3}-k$. Given $S_{k}$, the conditional expectation of $\mathcal{P}_{\left(a_{k+1}, b_{k+1}, c_{k+1}\right)}$ is

$$
\mathbb{E}\left[\mathcal{P}_{\left(a_{k+1}, b_{k+1}, c_{k+1}\right)} \mid S_{k}\right]=\frac{\mathcal{P}_{S_{k}^{c}}}{m_{k}} .
$$

For $k=1, \ldots, n$, define martingale differences

$$
\mathcal{D}_{k}=d_{1} d_{2} d_{3}\left(m_{n} / m_{k}\right) \mathcal{Q}_{\boldsymbol{T}}\left(\mathcal{P}_{\left(a_{k}, b_{k}, c_{k}\right)}-\mathcal{P}_{S_{k-1}^{c}} / m_{k-1}\right) \mathcal{Q}_{\boldsymbol{T}}
$$

Because $\mathcal{P}_{S_{0}^{c}}=\mathcal{I}$ and $S_{n}=\Omega$, we have

$$
\mathcal{Q}_{\boldsymbol{T}} \mathcal{P}_{\Omega} \mathcal{Q}_{\boldsymbol{T}} / m_{n}=\frac{\mathcal{D}_{n}}{d_{1} d_{2} d_{3} m_{n}}+\mathcal{Q}_{\boldsymbol{T}}\left(\mathcal{P}_{S_{n-1}^{c}} / m_{n-1}\right) \mathcal{Q}_{\boldsymbol{T}} / m_{n}+\mathcal{Q}_{\boldsymbol{T}} \mathcal{P}_{S_{n-1}} \mathcal{Q}_{\boldsymbol{T}} / m_{n}
$$




$$
\begin{aligned}
& =\frac{\mathcal{D}_{n}}{d_{1} d_{2} d_{3} m_{n}}+\mathcal{Q}_{\boldsymbol{T}}\left(1 / m_{n}-1 / m_{n-1}\right)+\mathcal{Q}_{\boldsymbol{T}} \mathcal{P}_{S_{n-1}} \mathcal{Q}_{\boldsymbol{T}} / m_{n-1} \\
& =\sum_{k=1}^{n} \frac{\mathcal{D}_{k}}{d_{1} d_{2} d_{3} m_{n}}+\mathcal{Q}_{\boldsymbol{T}}\left(1 / m_{n}-1 / m_{0}\right) .
\end{aligned}
$$

Since $1 / m_{n}-1 / m_{0}=n /\left(d_{1} d_{2} d_{3} m_{n}\right)$, it follows that

$$
\mathcal{Q}_{\boldsymbol{T}}\left(d_{1} d_{2} d_{3} / n\right) \mathcal{P}_{\Omega} \mathcal{Q}_{\boldsymbol{T}}-\mathcal{Q}_{\boldsymbol{T}}=\frac{1}{n} \sum_{k=1}^{n} \mathcal{D}_{k}
$$

Now an application of the matrix martingale Bernstein inequality (see, e.g., Tropp, 2011) gives

$$
\mathbb{P}\left\{\frac{1}{n}\left\|\sum_{k=1}^{n} \mathcal{D}_{k}\right\|>\tau\right\} \leq 2 \operatorname{rank}\left(\mathcal{Q}_{\boldsymbol{T}}\right) \exp \left(\frac{-n^{2} \tau^{2} / 2}{\sigma^{2}+n \tau M / 3}\right),
$$

where $M$ is a constant upper bound of $\left\|\mathcal{D}_{k}\right\|$ and $\sigma^{2}$ is a constant upper bound of

$$
\left\|\sum_{k=1}^{n} \mathbb{E}\left[\mathcal{D}_{k} \mathcal{D}_{k} \mid S_{k-1}\right]\right\|
$$

Note that $D_{k}$ are random self-adjoint operators.

Recall that $\mathcal{Q}_{\boldsymbol{T}}$ can be decomposed as a sum of orthogonal projections

$$
\begin{aligned}
\mathcal{Q}_{\boldsymbol{T}} & =\left(\mathcal{Q}_{\boldsymbol{T}}^{0}+\mathcal{Q}_{\boldsymbol{T}}^{1}\right)+\mathcal{Q}_{\boldsymbol{T}}^{2}+\mathcal{Q}_{\boldsymbol{T}}^{3} \\
& =\boldsymbol{I} \otimes \boldsymbol{P}_{\boldsymbol{T}}^{2} \otimes \boldsymbol{P}_{\boldsymbol{T}}^{3}+\boldsymbol{P}_{\boldsymbol{T}}^{1} \otimes \boldsymbol{P}_{\boldsymbol{T}^{\perp}}^{2} \otimes \boldsymbol{P}_{\boldsymbol{T}}^{3}+\boldsymbol{P}_{\boldsymbol{T}}^{1} \otimes \boldsymbol{P}_{\boldsymbol{T}}^{2} \otimes \boldsymbol{P}_{\boldsymbol{T}^{\perp}}^{3}
\end{aligned}
$$

The rank of $\mathcal{Q}_{\boldsymbol{T}}$, or equivalently the dimension of its range, is given by

$$
d_{1} r_{2} r_{3}+\left(d_{2}-r_{2}\right) r_{1} r_{3}+\left(d_{3}-r_{3}\right) r_{1} r_{2} \leq \bar{r}^{2} d .
$$

Hereafter, we shall write $r_{j}$ for $r_{j}(\boldsymbol{T}), \mu$ for $\mu(\boldsymbol{T})$, and $\bar{r}$ for $\bar{r}(\boldsymbol{T})$ for brevity when no confusion occurs. Since $\mathbb{E}\left[\mathcal{D}_{k} \mid S_{k-1}\right]=0$, the total variation is bounded by

$$
\begin{aligned}
& \max _{\left\|\mathcal{Q}_{\boldsymbol{T}} \boldsymbol{X}\right\|_{\mathrm{HS}}=1} \sum_{k=1}^{n} \mathbb{E}\left[\left\langle\mathcal{D}_{k} \boldsymbol{X}, \mathcal{D}_{k} \boldsymbol{X}\right\rangle \mid S_{k-1}\right] \\
\leq & \max _{\left\|\mathcal{Q}_{\boldsymbol{T}} \boldsymbol{X}\right\|_{\mathrm{HS}}=1} \sum_{k=1}^{n}\left(d_{1} d_{2} d_{3}\left(m_{n} / m_{k}\right)\right)^{2} \mathbb{E}\left[\left\langle\left(\mathcal{Q}_{\boldsymbol{T}} \mathcal{P}_{\left(a_{k}, b_{k}, c_{k}\right)} \mathcal{Q}_{\boldsymbol{T}}\right)^{2} \boldsymbol{X}, \boldsymbol{X}\right\rangle \mid S_{k-1}\right] \\
\leq & \sum_{k=1}^{n}\left(d_{1} d_{2} d_{3}\left(m_{n} / m_{k}\right)\right)^{2} m_{k-1}^{-1} \max _{\left\|\mathcal{Q}_{\boldsymbol{T}} \boldsymbol{X}\right\|_{\mathrm{HS}}=1} \sum_{a, b, c}\left\langle\left(\mathcal{Q}_{\boldsymbol{T}} \mathcal{P}_{(a, b, c)} \mathcal{Q}_{\boldsymbol{T}}\right)^{2} \boldsymbol{X}, \boldsymbol{X}\right\rangle .
\end{aligned}
$$

Since $m_{n} \leq m_{k}$ and $\sum_{k=1}^{n}\left(m_{n} / m_{k}\right) / m_{k-1}=n / d_{1} d_{2} d_{3}$,

$$
\max _{\left\|\mathcal{Q}_{\boldsymbol{T}} \boldsymbol{X}\right\|_{\mathrm{HS}}=1} \sum_{k=1}^{n} \mathbb{E}\left[\left\langle\mathcal{D}_{k} \boldsymbol{X}, \mathcal{D}_{k} \boldsymbol{X}\right\rangle \mid S_{k-1}\right] \leq n d_{1} d_{2} d_{3} \max _{a, b, c}\left\|\mathcal{Q}_{\boldsymbol{T}} \mathcal{P}_{(a, b, c)} \mathcal{Q}_{\boldsymbol{T}}\right\| .
$$


It then follows that

$$
\begin{aligned}
\max _{a, b, c}\left\|\mathcal{Q}_{\boldsymbol{T}} \mathcal{P}_{(a, b, c)} \mathcal{Q}_{\boldsymbol{T}}\right\| & =\max _{\left\|\mathcal{Q}_{\boldsymbol{T}} \boldsymbol{X}\right\|_{\mathrm{HS}}=1}\left\langle\mathcal{Q}_{\boldsymbol{T}} \mathcal{P}_{(a, b, c)} \mathcal{Q}_{\boldsymbol{T}} \boldsymbol{X}, \mathcal{Q}_{\boldsymbol{T}} \boldsymbol{X}\right\rangle \\
& =\max _{\left\|\mathcal{Q}_{\boldsymbol{T}} \boldsymbol{X}\right\|_{\mathrm{HS}}=1}\left\langle\mathcal{Q}_{\boldsymbol{T}} \boldsymbol{e}_{a} \otimes \boldsymbol{e}_{b} \otimes \boldsymbol{e}_{c}, \mathcal{Q}_{\boldsymbol{T}} \boldsymbol{X}\right\rangle^{2} \\
& \leq \frac{\mu^{2} \bar{r}^{2} d}{d_{1} d_{2} d_{3}}
\end{aligned}
$$

Consequently, we may take $\sigma^{2}=n \mu_{0}^{2} \bar{r}^{2} d$. Similarly,

$$
M \leq \max _{k} d_{1} d_{2} d_{3}\left(m_{n} / m_{k}\right) 2 \max _{a, b, c}\left\|\mathcal{Q}_{\boldsymbol{T}} \mathcal{P}_{(a, b, c)} \mathcal{Q}_{\boldsymbol{T}}\right\| \leq 2 \mu^{2} \bar{r}^{2} d
$$

Inserting the expression and bounds for $\operatorname{rank}\left(\mathcal{Q}_{\boldsymbol{T}}\right), \sigma^{2}$ and $M$ into the Bernstein inequality, we find

$$
\mathbb{P}\left\{\frac{1}{n}\left\|\sum_{k=1}^{n} \mathcal{D}_{k}\right\|>\tau\right\} \leq 2\left(\bar{r}^{2} d\right) \exp \left(\frac{-\tau^{2} / 2}{1+2 \tau / 3}\left(\frac{n}{\mu^{2} \bar{r}^{2} d}\right)\right),
$$

which completes the proof because $\mu(\boldsymbol{T}) \leq \mu_{0}$ and $\bar{r}(\boldsymbol{T})=r$.

\subsection{Proof of Lemma 6.}

In proving Lemma 6, we consider first (12). Let $\boldsymbol{X}$ be a tensor with $\|\boldsymbol{X}\|_{\max } \leq 1$. Similar to before, write

$$
\mathcal{D}_{i}=d_{1} d_{2} d_{3} \mathcal{Q}_{\boldsymbol{T}} \mathcal{P}_{\left(a_{i}, b_{i}, c_{i}\right)}-\mathcal{Q}_{\boldsymbol{T}}
$$

for $i=1, \ldots, n_{1}$. Again, we shall also write $\mu$ for $\mu(\boldsymbol{T})$, and $\bar{r}$ for $\bar{r}(\boldsymbol{T})$ for brevity. Observe that for each point $(a, b, c) \in\left[d_{1}\right] \times\left[d_{2}\right] \times\left[d_{3}\right]$,

$$
\begin{aligned}
& \frac{1}{d_{1} d_{2} d_{3}}\left|\left\langle\boldsymbol{e}_{a} \otimes \boldsymbol{e}_{b} \otimes \boldsymbol{e}_{c}, \mathcal{D}_{i} \boldsymbol{X}\right\rangle\right| \\
= & \left|\left\langle\mathcal{Q}_{\boldsymbol{T}}\left(\boldsymbol{e}_{a} \otimes \boldsymbol{e}_{b} \otimes \boldsymbol{e}_{c}\right), \mathcal{Q}_{\boldsymbol{T}}\left(\boldsymbol{e}_{a_{k}} \otimes \boldsymbol{e}_{b_{k}} \otimes \boldsymbol{e}_{c_{k}}\right)\right\rangle X\left(a_{k}, b_{k}, c_{k}\right)-\left\langle\mathcal{Q}_{\boldsymbol{T}}\left(\boldsymbol{e}_{a} \otimes \boldsymbol{e}_{b} \otimes \boldsymbol{e}_{c}\right), \mathcal{Q}_{\boldsymbol{T}} \boldsymbol{X}\right\rangle /\left(d_{1} d_{2} d_{3}\right)\right| \\
\leq & 2 \max _{a, b, c}\left\|\mathcal{Q}_{\boldsymbol{T}}\left(\boldsymbol{e}_{a} \otimes \boldsymbol{e}_{b} \otimes \boldsymbol{e}_{c}\right)\right\|_{\mathrm{HS}}^{2}\|\boldsymbol{X}\|_{\max } \\
\leq & 2 \mu^{2} \bar{r}^{2} d /\left(d_{1} d_{2} d_{3}\right) .
\end{aligned}
$$

Since the variance of a variable is no greater than the second moment,

$$
\begin{aligned}
& \mathbb{E}\left(\frac{1}{d_{1} d_{2} d_{3}}\left|\left\langle\boldsymbol{e}_{a} \otimes \boldsymbol{e}_{b} \otimes \boldsymbol{e}_{c}, \mathcal{Q}_{\boldsymbol{T}} \mathcal{D}_{i} \boldsymbol{X}\right\rangle\right|\right)^{2} \\
\leq & \mathbb{E}\left|\left\langle\mathcal{Q}_{\boldsymbol{T}}\left(\boldsymbol{e}_{a} \otimes \boldsymbol{e}_{b} \otimes \boldsymbol{e}_{c}\right), \mathcal{Q}_{\boldsymbol{T}}\left(\boldsymbol{e}_{a^{\prime}} \otimes \boldsymbol{e}_{b^{\prime}} \otimes \boldsymbol{e}_{c^{\prime}}\right)\right\rangle X\left(a_{k}, b_{k}, c_{k}\right)\right|^{2} \\
\leq & \frac{1}{d_{1} d_{2} d_{3}} \sum_{a^{\prime}, b^{\prime}, c^{\prime}}\left|\left\langle\mathcal{Q}_{\boldsymbol{T}} \boldsymbol{e}_{a} \otimes \boldsymbol{e}_{b} \otimes \boldsymbol{e}_{c}, \mathcal{Q}_{\boldsymbol{T}}\left(\boldsymbol{e}_{a^{\prime}} \otimes \boldsymbol{e}_{b^{\prime}} \otimes \boldsymbol{e}_{c^{\prime}}\right)\right\rangle\right|^{2} \\
= & \frac{1}{d_{1} d_{2} d_{3}}\left\|\mathcal{Q}_{\boldsymbol{T}}\left(\boldsymbol{e}_{a} \otimes \boldsymbol{e}_{b} \otimes \boldsymbol{e}_{c}\right)\right\|_{\mathrm{HS}}^{2}
\end{aligned}
$$




$$
\leq \mu^{2} \bar{r}^{2} d /\left(d_{1} d_{2} d_{3}\right)^{2} .
$$

Since $\left\langle\boldsymbol{e}_{a} \otimes \boldsymbol{e}_{b} \otimes \boldsymbol{e}_{c}, \mathcal{Q}_{\boldsymbol{T}} \mathcal{D}_{i} \boldsymbol{X}\right\rangle$ are iid random variables, the Bernstein inequality yields

$$
\mathbb{P}\left\{\left|\frac{1}{n_{1}} \sum_{i=1}^{n_{1}}\left\langle\boldsymbol{e}_{a} \otimes \boldsymbol{e}_{b} \otimes \boldsymbol{e}_{c}, \mathcal{Q}_{\boldsymbol{T}} \mathcal{D}_{i} \boldsymbol{X}\right\rangle\right|>\tau\right\} \leq 2 \exp \left(-\frac{\left(n_{1} \tau\right)^{2} / 2}{n_{1} \mu^{2} \bar{r}^{2} d+n_{1} \tau 2 \mu^{2} \bar{r}^{2} d / 3}\right) .
$$

This yields (12) by the union bound.

The proof of (11) is similar, but the matrix Bernstein inequality is used. We equip $\mathbb{R}^{d_{1} \times d_{2} \times d_{3}}$ with the Hilbert-Schmidt norm so that it can be viewed as the Euclidean space. As linear maps in this Euclidean space, the operators $D_{i}$ are just random matrices. Since the projection $\mathcal{P}_{(a, b, c)}: \boldsymbol{X} \rightarrow\left\langle\boldsymbol{e}_{a} \otimes \boldsymbol{e}_{b} \otimes \boldsymbol{e}_{c}, \boldsymbol{X}\right\rangle \boldsymbol{e}_{a} \otimes \boldsymbol{e}_{b} \otimes \boldsymbol{e}_{c}$ is of rank 1,

$$
\left\|\mathcal{Q}_{\boldsymbol{T}} \mathcal{P}_{(a, b, c)} \mathcal{Q}_{\boldsymbol{T}}\right\|=\left\|\mathcal{Q}_{\boldsymbol{T}}\left(\boldsymbol{e}_{a} \otimes \boldsymbol{e}_{b} \otimes \boldsymbol{e}_{c}\right)\right\|_{\mathrm{HS}}^{2} \leq \mu^{2} \bar{r}^{2} d /\left(d_{1} d_{2} d_{3}\right) .
$$

It follows that $\left\|\mathcal{D}_{i}\right\| \leq 2 \mu^{2} \bar{r}^{2} d$. Moreover, $\mathcal{D}_{i}$ is a self-adjoint operator and its covariance operator is bounded by

$$
\begin{aligned}
\max _{\|\boldsymbol{X}\|_{\mathrm{HS}}=1} \mathbb{E}\left\|\mathcal{D}_{i} \boldsymbol{X}\right\|_{\mathrm{HS}}^{2} & \leq\left(d_{1} d_{2} d_{3}\right)^{2} \max _{\|\boldsymbol{X}\|_{\mathrm{HS}}=1} \mathbb{E}\left\langle\left(\mathcal{Q}_{\boldsymbol{T}} \mathcal{P}_{\left(a_{k}, b_{k}, c_{k}\right)} \mathcal{Q}_{\boldsymbol{T}}\right)^{2} \boldsymbol{X}, \boldsymbol{X}\right\rangle \\
& \leq d_{1} d_{2} d_{3} \sum_{a, b, c}\left\|\mathcal{Q}_{\boldsymbol{T}}\left(\boldsymbol{e}_{a} \otimes \boldsymbol{e}_{b} \otimes \boldsymbol{e}_{c}\right)\right\|_{\mathrm{HS}}^{2}\left\langle\boldsymbol{e}_{a} \otimes \boldsymbol{e}_{b} \otimes \boldsymbol{e}_{c}, \boldsymbol{X}\right\rangle^{2} \\
& \leq d_{1} d_{2} d_{3} \max _{a, b, c}\left\|\mathcal{Q}_{\boldsymbol{T}}\left(\boldsymbol{e}_{a} \otimes \boldsymbol{e}_{b} \otimes \boldsymbol{e}_{c}\right)\right\|_{\mathrm{HS}}^{2} \\
& =\mu^{2} \bar{r}^{2} d
\end{aligned}
$$

Consequently, by the matrix Bernstein inequality (Tropp, 2011),

$$
\mathbb{P}\left\{\left\|\frac{1}{n_{1}} \sum_{i=1}^{n_{1}} \mathcal{D}_{i}\right\|>\tau\right\} \leq 2 \operatorname{rank}\left(\mathcal{Q}_{\boldsymbol{T}}\right) \exp \left(\frac{\tau^{2} / 2}{1+2 \tau / 3}\left(\frac{n_{1}}{\mu^{2} \bar{r}^{2} d}\right)\right) .
$$

This completes the proof due to the fact that $\operatorname{rank}\left(\mathcal{Q}_{\boldsymbol{T}}\right) \leq \bar{r}^{2} d$.

\section{Concentration Inequalities for Sparse Tensors}

We now derive probabilistic bounds for $\left\|\mathcal{R}_{\ell} \boldsymbol{X}\right\|$ when $\left(a_{i}, b_{i}, c_{i}\right)$ s are iid vectors uniformly sampled from $\left[d_{1}\right] \times\left[d_{2}\right] \times\left[d_{3}\right]$ and $\boldsymbol{X}=\mathcal{Q}_{\boldsymbol{T}} \boldsymbol{X}$ with small $\|\boldsymbol{X}\|_{\max }$.

\subsection{Symmetrization}

We are interested in bounding

$$
\max _{\boldsymbol{X} \in \mathscr{U}(\eta)} \mathbb{P}\left\{\left\|\frac{d_{1} d_{2} d_{3}}{n} \sum_{i=1}^{n} \mathcal{P}_{\left(a_{i}, b_{i}, c_{i}\right)} \boldsymbol{X}-\boldsymbol{X}\right\| \geq t\right\},
$$


e.g. with $(n, \eta, t)$ replaced by $\left(n_{1},\left(\alpha_{0} \sqrt{r}\right) \wedge \sqrt{d_{1} d_{2} d_{3}}, 1 / 8\right)$ in the proof of Lemma 7 , where

$$
\mathscr{U}(\eta)=\left\{\boldsymbol{X}: \mathcal{Q}_{\boldsymbol{T}} \boldsymbol{X}=\boldsymbol{X},\|\boldsymbol{X}\|_{\max } \leq \eta / \sqrt{d_{1} d_{2} d_{3}}\right\}
$$

Our first step is symmetrization.

Lemma 8 Let $\epsilon_{i}$ s be a Rademacher sequence, that is a sequence of i.i.d. $\epsilon_{i}$ with $\mathbb{P}\left\{\epsilon_{i}=1\right\}=$ $\mathbb{P}\left\{\epsilon_{i}=-1\right\}=1 / 2$. Then

$$
\begin{aligned}
\max _{\boldsymbol{X} \in \mathscr{U}(\eta)} \mathbb{P}\left\{\left\|\frac{d_{1} d_{2} d_{3}}{n} \sum_{i=1}^{n} \mathcal{P}_{\left(a_{i}, b_{i}, c_{i}\right)} \boldsymbol{X}-\boldsymbol{X}\right\| \geq t\right\} \\
\leq 4 \max _{\boldsymbol{X} \in \mathscr{U}(\eta)} \mathbb{P}\left\{\left\|\frac{d_{1} d_{2} d_{3}}{n} \sum_{i=1}^{n} \epsilon_{i} \mathcal{P}_{\left(a_{i}, b_{i}, c_{i}\right)} \boldsymbol{X}\right\| \geq t / 2\right\}+4 \exp \left(-\frac{n t^{2} / 2}{\eta^{2}+2 \eta t \sqrt{d_{1} d_{2} d_{3}} / 3}\right) .
\end{aligned}
$$

Proof of Lemma 8. The standard symmetrization argument gives

$$
\begin{aligned}
\max _{\boldsymbol{X} \in \mathscr{U}(\eta)} \mathbb{P}\left\{\left\|\frac{d_{1} d_{2} d_{3}}{n} \sum_{i=1}^{n} \mathcal{P}_{\left(a_{i}, b_{i}, c_{i}\right)} \boldsymbol{X}-\boldsymbol{X}\right\| \geq t\right\} \\
\leq 4 \max _{\boldsymbol{X} \in \mathscr{U}(\eta)} \mathbb{P}\left\{\left\|\frac{d_{1} d_{2} d_{3}}{n} \sum_{i=1}^{n} \epsilon_{i} \mathcal{P}_{\left(a_{i}, b_{i}, c_{i}\right)} \boldsymbol{X}\right\| \geq t / 2\right\} \\
+2 \max _{\boldsymbol{X} \in \mathscr{U}(\eta)} \max _{\|\boldsymbol{u}\|=\|\boldsymbol{v}\|=\|\boldsymbol{w}\|=1} \mathbb{P}\left\{\left\langle\boldsymbol{u} \otimes \boldsymbol{v} \otimes \boldsymbol{w}, \frac{d_{1} d_{2} d_{3}}{n} \sum_{i=1}^{n} \mathcal{P}_{\left(a_{i}, b_{i}, c_{i}\right)} \boldsymbol{X}-\boldsymbol{X}\right\rangle>t\right\} .
\end{aligned}
$$

It remains to bound the second quantity on the right-hand side. To this end, denote by

$$
\xi_{i}=\left\langle\boldsymbol{u} \otimes \boldsymbol{v} \otimes \boldsymbol{w}, d_{1} d_{2} d_{3} \mathcal{P}_{\left(a_{i}, b_{i}, c_{i}\right)} \boldsymbol{X}-\boldsymbol{X}\right\rangle
$$

For $\|\boldsymbol{u}\|=\|\boldsymbol{v}\|=\|\boldsymbol{w}\|=1$ and $\boldsymbol{X} \in \mathscr{U}(\eta), \xi_{i}$ are iid variables with $\mathbb{E} \xi_{i}=0$, $\left|\xi_{i}\right| \leq$ $2 d_{1} d_{2} d_{3}\|\boldsymbol{X}\|_{\max } \leq 2 \eta \sqrt{d_{1} d_{2} d_{3}}$ and $\mathbb{E} \xi_{i}^{2} \leq\left(d_{1} d_{2} d_{3}\right)\|\boldsymbol{X}\|_{\max }^{2} \leq \eta^{2}$. Thus, the statement follows from the Bernstein inequality.

In the light of Lemma 8, it suffices to consider bounding

$$
\max _{\boldsymbol{X} \in \mathscr{U}(\eta)} \mathbb{P}\left\{\left\|\frac{d_{1} d_{2} d_{3}}{n} \sum_{i=1}^{n} \epsilon_{i} \mathcal{P}_{\left(a_{i}, b_{i}, c_{i}\right)} \boldsymbol{X}\right\| \geq t / 2\right\}
$$

To this end, we use a thinning method to control the spectral norm of tensors. 


\subsection{Thinning of the spectral norm of tensors}

Recall that the spectral norm of a tensor $\boldsymbol{Z} \in \mathbb{R}^{d_{1} \times d_{2} \times d_{3}}$ is defined as

$$
\|\boldsymbol{Z}\|=\max _{\substack{\boldsymbol{u} \in \mathbb{R}^{d_{1}}, \boldsymbol{v} \in \mathbb{R}^{d_{2}}, \boldsymbol{w} \in \mathbb{R}^{d_{3}} \\\|\boldsymbol{u}\| \vee\|\boldsymbol{v}\| \vee\|\boldsymbol{w}\| \leq 1}}\langle\boldsymbol{u} \otimes \boldsymbol{v} \otimes \boldsymbol{w}, \boldsymbol{Z}\rangle .
$$

We first use a thinning method to discretize maximization in the unit ball in $\mathbb{R}^{d_{j}}$ to the problem involving only vectors taking values 0 or $\pm 2^{-\ell / 2}, \ell \leq m_{j}:=\left\lceil\log _{2} d_{j}\right\rceil$, that is, binary "digitalized" vectors that belong to

$$
\mathscr{B}_{m_{j}, d_{j}}=\left\{0, \pm 1, \pm 2^{-1 / 2}, \ldots, \pm 2^{-m_{j} / 2}\right\}^{d} \cap\left\{\boldsymbol{u} \in \mathbb{R}^{d_{j}}:\|\boldsymbol{u}\| \leq 1\right\}
$$

Lemma 9 For any tensor $\boldsymbol{Z} \in \mathbb{R}^{d_{1} \times d_{2} \times d_{3}}$,

$$
\|\boldsymbol{Z}\| \leq 8 \max \left\{\langle\boldsymbol{u} \otimes \boldsymbol{v} \otimes \boldsymbol{w}, \boldsymbol{Z}\rangle: \boldsymbol{u} \in \mathscr{B}_{m_{1}, d_{1}}, \boldsymbol{v} \in \mathscr{B}_{m_{2}, d_{2}}, \boldsymbol{w} \in \mathscr{B}_{m_{3}, d_{3}}\right\},
$$

where $m_{j}:=\left\lceil\log _{2} d_{j}\right\rceil, j=1,2,3$.

Proof of Lemma 9, Denote by

$$
C_{m, d}=\min _{\|\boldsymbol{a}\|=1} \max _{u \in \mathscr{B}_{m, d}} \boldsymbol{u}^{\top} \boldsymbol{a}
$$

which bounds the effect of discretization. Let $\boldsymbol{X}$ be a linear mapping from $\mathbb{R}^{d}$ to a linear space equipped with a seminorm $\|\cdot\|$. Then, $\|\boldsymbol{X} \boldsymbol{u}\|$ can be written as the maximum of $\phi(\boldsymbol{X} \boldsymbol{u})$ over linear functionals $\phi(\cdot)$ of unit dual norm. Since $\max _{\|\boldsymbol{u}\| \leq 1} \boldsymbol{u}^{\top} \boldsymbol{a}=1$ for $\|\boldsymbol{a}\|=1$, it follows from the definition of $C_{m, d}$ that

$$
\max _{\|\boldsymbol{u}\| \leq 1} \boldsymbol{u}^{\top} \boldsymbol{a} \leq\|\boldsymbol{a}\| C_{m, d}^{-1} \max _{\boldsymbol{u} \in \mathscr{B}_{m, d}} \boldsymbol{u}^{\top}(\boldsymbol{a} /\|\boldsymbol{a}\|)=C_{m, d}^{-1} \max _{\boldsymbol{u} \in \mathscr{B}_{m, d}} \boldsymbol{u}^{\top} \boldsymbol{a}
$$

for every $\boldsymbol{a} \in \mathbb{R}^{d}$ with $\|\boldsymbol{a}\|>0$. Consequently, for any positive integer $m$,

$$
\max _{\|\boldsymbol{u}\| \leq 1}\|\boldsymbol{X} \boldsymbol{u}\|=\max _{\boldsymbol{a}: \boldsymbol{a}^{\top} \boldsymbol{v}=\phi(\boldsymbol{X} \boldsymbol{v}) \forall \boldsymbol{v}\|\boldsymbol{u}\| \leq 1} \boldsymbol{a}^{\top} \boldsymbol{u} \leq C_{m, d}^{-1} \max _{\boldsymbol{u} \in \mathscr{B}_{m, d}}\|\boldsymbol{X} \boldsymbol{u}\| .
$$

An application of the above inequality to each coordinate yields

$$
\|\boldsymbol{Z}\| \leq C_{m_{1}, d_{1}}^{-1} C_{m_{2}, d_{2}}^{-1} C_{m_{3}, d_{3}}^{-1} \max _{\boldsymbol{u} \in \mathscr{B}_{m_{1}, d_{1}}, \boldsymbol{v} \in \mathscr{B}_{m_{2}, d_{2}}, \boldsymbol{w} \in \mathscr{B}_{m_{3}, d_{3}}}\langle\boldsymbol{u} \otimes \boldsymbol{v} \otimes \boldsymbol{w}, \boldsymbol{Z}\rangle
$$

It remains to show that $C_{m_{j}, d_{j}} \geq 1 / 2$. To this end, we prove a stronger result that for any $m$ and $d$,

$$
C_{m, d}^{-1} \leq \sqrt{2+2(d-1) /\left(2^{m}-1\right)}
$$


Consider first a continuous version of $C_{m, d}$ :

$$
C_{m, d}^{\prime}=\min _{\|\boldsymbol{a}\|=1} \max _{\boldsymbol{u} \in \mathscr{B}_{m, d}^{\prime}} \boldsymbol{a}^{\top} \boldsymbol{u}
$$

where $\mathscr{B}_{m, d}^{\prime}=\left\{t: t^{2} \in[0,1] \backslash\left(0,2^{-m}\right)\right\}^{d} \cap\{\boldsymbol{u}:\|\boldsymbol{u}\| \leq 1\}$. Without loss of generality, we confine the calculation to nonnegative ordered $\boldsymbol{a}=\left(a_{1}, \ldots, a_{d}\right)^{\top}$ satisfying $0 \leq a_{1} \leq \ldots \leq a_{d}$ and $\|\boldsymbol{a}\|=1$. Let

$$
k=\max \left\{j: 2^{m} a_{j}^{2}+\sum_{i=1}^{j-1} a_{i}^{2} \leq 1\right\} \quad \text { and } \quad \boldsymbol{v}=\frac{\left(a_{i} I\{i>k\}\right)_{d \times 1}}{\left\{1-\sum_{i=1}^{k} a_{i}^{2}\right\}^{1 / 2}} .
$$

Because $2^{m} v_{k+1}^{2}=2^{m} a_{k+1}^{2} /\left(1-\sum_{i=1}^{k} a_{i}^{2}\right) \geq 1$, we have $\boldsymbol{v} \in \mathscr{B}_{m, d}^{\prime}$. By the definition of $k$, there exists $x^{2} \geq a_{k}^{2}$ satisfying

$$
\left(2^{m}-1\right) x^{2}+\sum_{i=1}^{k} a_{i}^{2}=1
$$

It follows that

$$
\sum_{i=1}^{k} a_{i}^{2}=\frac{\sum_{i=1}^{k} a_{i}^{2}}{\left(2^{m}-1\right) x^{2}+\sum_{i=1}^{k} a_{i}^{2}} \leq \frac{k x^{2}}{\left(2^{m}-1\right) x^{2}+k x^{2}} \leq \frac{d-1}{2^{m}+d-2} .
$$

Because $\boldsymbol{a}^{\top} \boldsymbol{v}=\left(1-\sum_{i=1}^{k} a_{i}^{2}\right)^{1 / 2}$ for this specific $\boldsymbol{v} \in \mathscr{B}_{m, d}^{\prime}$, we get

$$
C_{m, d}^{\prime} \geq \min _{\|a\|_{2}=1}\left(1-\sum_{i=1}^{k} a_{i}^{2}\right)^{1 / 2} \geq\left(1-\frac{d-1}{2^{m}+d-2}\right)^{1 / 2}=\left(\frac{2^{m}-1}{2^{m}+d-2}\right)^{1 / 2} .
$$

Now because every $\boldsymbol{v} \in \mathscr{B}_{m, d}^{\prime}$ with nonnegative components matches a $\boldsymbol{u} \in \mathscr{B}_{m, d}$ with

$$
\operatorname{sgn}\left(v_{i}\right) \sqrt{2} u_{i} \geq\left|v_{i}\right| \geq \operatorname{sgn}\left(v_{i}\right) u_{i},
$$

we find $C_{m, d} \geq C_{m, d}^{\prime} / \sqrt{2}$. Consequently,

$$
1 / C_{m, d} \leq \sqrt{2} / C_{m, d}^{\prime} \leq \sqrt{2}\left\{1+(d-1) /\left(2^{m}-1\right)\right\}^{1 / 2} .
$$

It follows from Lemma 9 that the spectrum norm $\|\boldsymbol{Z}\|$ is of the same order as the maximum of $\langle\boldsymbol{u} \otimes \boldsymbol{v} \otimes \boldsymbol{w}, \boldsymbol{Z}\rangle$ over $\boldsymbol{u} \in \mathscr{B}_{m_{1}, d_{1}}, \boldsymbol{v} \in \mathscr{B}_{m_{2}, d_{2}}$ and $\boldsymbol{w} \in \mathscr{B}_{m_{3}, d_{3}}$. We will further decompose such tensors $\boldsymbol{u} \otimes \boldsymbol{v} \otimes \boldsymbol{w}$ according to the absolute value of their entries and bound the entropy of the components in this decomposition. 


\subsection{Spectral norm of tensors with sparse support}

Denote by $D_{j}$ a "digitalization" operator such that $D_{j}(\boldsymbol{X})$ will zero out all entries of $\boldsymbol{X}$ whose absolute value is not $2^{-j / 2}$, that is

$$
D_{j}(\boldsymbol{X})=\sum_{a, b, c} \mathbb{I}\left\{\left|\left\langle\boldsymbol{e}_{a} \otimes \boldsymbol{e}_{b} \otimes \boldsymbol{e}_{c}, \boldsymbol{X}\right\rangle\right|=2^{-j / 2}\right\}\left\langle\boldsymbol{e}_{a} \otimes \boldsymbol{e}_{b} \otimes \boldsymbol{e}_{c}, \boldsymbol{X}\right\rangle \boldsymbol{e}_{a} \otimes \boldsymbol{e}_{b} \otimes \boldsymbol{e}_{c} .
$$

With this notation, it is clear that for $\boldsymbol{u} \in \mathscr{B}_{m_{1}, d_{1}}, \boldsymbol{v} \in \mathscr{B}_{m_{2}, d_{2}}$ and $\boldsymbol{w} \in \mathscr{B}_{m_{3}, d_{3}}$,

$$
\langle\boldsymbol{u} \otimes \boldsymbol{v} \otimes \boldsymbol{w}, \boldsymbol{X}\rangle=\sum_{j=0}^{m_{1}+m_{2}+m_{3}}\left\langle D_{j}(\boldsymbol{u} \otimes \boldsymbol{v} \otimes \boldsymbol{w}), \boldsymbol{X}\right\rangle .
$$

The possible choice of $D_{j}(\boldsymbol{u} \otimes \boldsymbol{v} \otimes \boldsymbol{w})$ in the above expression may be further reduced if $\boldsymbol{X}$ is sparse. More specifically, denote by

$$
\operatorname{supp}(\boldsymbol{X})=\left\{\omega \in\left[d_{1}\right] \times\left[d_{2}\right] \times\left[d_{3}\right]: X(\omega) \neq 0\right\} .
$$

Define the maximum aspect ratio of $\operatorname{supp}(\boldsymbol{X})$ as

$$
\nu_{\operatorname{supp}(\boldsymbol{X})}=\max _{\ell=1,2,3} \max _{i_{k}: k \neq \ell}\left|\left\{i_{\ell}:\left(i_{1}, i_{2}, i_{3}\right) \in \operatorname{supp}(\boldsymbol{X})\right\}\right| .
$$

In other words, the quantity $\nu_{\operatorname{Supp}(\boldsymbol{X})}$ is the maximum $\ell_{0}$ norm of the fibers of the third-order tensor. We observe first that, if $\operatorname{supp}(\boldsymbol{X})$ is a uniformly sampled subset of $\left[d_{1}\right] \times\left[d_{2}\right] \times\left[d_{3}\right]$, then it necessarily has a small aspect ratio.

Lemma 10 Let $\Omega$ be a uniformly sampled subset of $\left[d_{1}\right] \times\left[d_{2}\right] \times\left[d_{3}\right]$ without replacement. Let $d=d_{1}+d_{2}+d_{3}, p^{*}=\max \left(d_{1}, d_{2}, d_{3}\right) /\left(d_{1} d_{2} d_{3}\right)$, and $\nu_{1}=\left(d^{\delta_{1}} e n p^{*}\right) \vee\left\{(3+\beta) / \delta_{1}\right\}$ with a certain $\delta_{1} \in[1 / \log d, 1]$. Then,

$$
\mathbb{P}\left\{\nu_{\Omega} \geq \nu_{1}\right\} \leq d^{-\beta-1} / 3
$$

Proof of Lemma 10. Let $p_{1}=d_{1} /\left(d_{1} d_{2} d_{3}\right), t=\log \left(\nu_{1} /\left(n p^{*}\right)\right) \geq 1$, and

$$
N_{i_{2} i_{3}}=\left|\left\{i_{\ell}:\left(i_{1}, i_{2}, i_{3}\right) \in \Omega\right\}\right| .
$$

Because $N_{i_{2} i_{3}}$ follows the Hypergeometric $\left(d_{1} d_{2} d_{3}, d_{1}, n\right)$ distribution, its moment generating function is no greater than that of $\operatorname{Binomial}\left(n, p_{1}\right)$. Due to $p_{1} \leq p^{*}$,

$$
\mathbb{P}\left\{N_{i_{2} i_{3}} \geq \nu_{1}\right\} \leq \exp \left(-t \nu_{1}+n p^{*}\left(e^{t}-1\right)\right) \leq \exp \left(-\nu_{1} \log \left(\nu_{1} /\left(e n p^{*}\right)\right)\right) .
$$


The condition on $\nu_{1}$ implies $\nu_{1} \log \left(\nu_{1} /\left(e n p^{*}\right)\right) \geq(3+\beta) \log d$. By the union bound,

$$
\mathbb{P}\left\{\max _{i_{2}, i_{3}} N_{i_{2} i_{3}} \geq \nu_{1}\right\} \leq d_{2} d_{3} d^{-3-\beta} .
$$

By symmetry, the same tail probability bound also holds for $\max _{i_{1}, i_{3}}\left|\left\{i_{2}:\left(i_{1}, i_{2}, i_{3}\right) \in \Omega\right\}\right|$ and $\max _{i_{1}, i_{2}}\left|\left\{i_{3}:\left(i_{1}, i_{2}, i_{3}\right) \in \Omega\right\}\right|$, so that $\mathbb{P}\left\{\nu_{\Omega} \geq \nu_{1}\right\} \leq\left(d_{1} d_{2}+d_{1} d_{3}+d_{2} d_{3}\right) d^{-3-\beta}$. The conclusion follows from $d_{1} d_{2}+d_{1} d_{3}+d_{2} d_{3} \leq d^{2} / 3$.

We are now in position to further reduce the set of maximization in defining the spectrum norm of sparse tensors. To this end, denote for a block $A \times B \times C \subseteq\left[d_{1}\right] \times\left[d_{2}\right] \times\left[d_{3}\right]$,

$$
h(A \times B \times C)=\min \{\nu:|A| \leq \nu|B||C|,|B| \leq \nu|A||C|,|C| \leq \nu|A||B|\} .
$$

It is clear that for any block $A \times B \times C$, there exists $\widetilde{A} \subseteq A, \widetilde{B} \subseteq B$ and $\widetilde{C} \subseteq C$ such that $h(\widetilde{A} \times \widetilde{B} \times \widetilde{C}) \leq \nu_{\Omega}$ and

$$
(A \times B \times C) \cap \Omega=(\widetilde{A} \times \widetilde{B} \times \widetilde{C}) \cap \Omega .
$$

For $\boldsymbol{u} \in \mathscr{B}_{m_{1}, d_{1}}, \boldsymbol{v} \in \mathscr{B}_{m_{2}, d_{2}}$ and $\boldsymbol{w} \in \mathscr{B}_{m_{3}, d_{3}}$, let $A_{i_{1}}=\left\{a: u_{a}^{2}=2^{-i_{1}}\right\}, B_{i_{2}}=\left\{b: v_{b}^{2}=2^{-i_{2}}\right\}$ and $C_{i_{3}}=\left\{c: w_{c}^{2}=2^{-i_{3}}\right\}$, and define

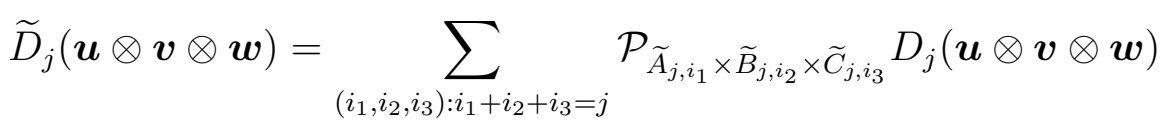

where $\widetilde{A}_{j, i_{1}} \subseteq A_{j, i_{1}}, \widetilde{B}_{j, i_{2}} \subseteq B_{j, i_{2}}$ and $\widetilde{C}_{j, i_{3}} \subseteq C_{j, i_{3}}$ satisfying $h\left(\widetilde{A}_{j, i_{1}} \times \widetilde{B}_{j, i_{2}} \times \widetilde{C}_{j, i_{3}}\right) \leq \nu_{\Omega}$ and

$$
\left(A_{i_{1}} \times B_{i_{2}} \times C_{i_{3}}\right) \cap \Omega=\left(\widetilde{A}_{j, i_{1}} \times \widetilde{B}_{j, i_{2}} \times \widetilde{C}_{j, i_{3}}\right) \cap \Omega .
$$

Because $\mathcal{P}_{\Omega} D_{j}(\boldsymbol{u} \otimes \boldsymbol{v} \otimes \boldsymbol{w})$ is supported in $\cup_{\left(i_{1}, i_{2}, i_{3}\right): i_{1}+i_{2}+i_{3}=j}\left(A_{i_{1}} \times B_{i_{2}} \times C_{i_{3}}\right) \cap \Omega$, we have

$$
\mathcal{P}_{\Omega} \widetilde{D}_{j}(\boldsymbol{u} \otimes \boldsymbol{v} \otimes \boldsymbol{w})=\mathcal{P}_{\Omega} D_{j}(\boldsymbol{u} \otimes \boldsymbol{v} \otimes \boldsymbol{w}) .
$$

This observation, together with Lemma 9, leads to the following characterization of the spectral norm of a tensor support on a set with bounded aspect ratio.

Lemma 11 Let $m_{\ell}=\left\lceil\log _{2} d_{\ell}\right\rceil$ for $\ell=1,2,3$, and $D_{j}(\cdot)$ and $\widetilde{D}_{j}(\cdot)$ be as in (17) and (20) respectively. Define

$$
\mathscr{B}_{\Omega, m_{*}}^{*}=\left\{\sum_{0 \leq j \leq m_{*}} \widetilde{D}_{j}\left(\boldsymbol{u}_{1} \otimes \boldsymbol{u}_{2} \otimes \boldsymbol{u}_{3}\right)+\sum_{m_{*}<j \leq m^{*}} D_{j}\left(\boldsymbol{u}_{1} \otimes \boldsymbol{u}_{2} \otimes \boldsymbol{u}_{3}\right): \boldsymbol{u}_{\ell} \in \mathscr{B}_{m_{\ell}, d_{\ell}}\right\},
$$

and $\mathscr{B}_{\nu, m_{*}}^{*}=\cup_{\nu_{\Omega} \leq \nu} \mathscr{B}_{\Omega, m_{*}}^{*}$. Let $\boldsymbol{X} \in \mathbb{R}^{d_{1} \times d_{2} \times d_{3}}$ be a tensor with $\operatorname{supp}(\boldsymbol{X}) \subseteq \Omega$. For any $0 \leq m_{*} \leq m_{1}+m_{2}+m_{3}$ and $\nu \geq \nu_{\Omega}$,

$$
\|\boldsymbol{X}\| \leq 8 \max _{\boldsymbol{Y} \in \mathscr{B}_{\Omega, m_{*}^{*}}^{*}}\langle\boldsymbol{Y}, \boldsymbol{X}\rangle \leq 8 \max _{\boldsymbol{Y} \in \mathscr{B}_{\nu, m_{*}^{*}}^{*}}\langle\boldsymbol{Y}, \boldsymbol{X}\rangle .
$$




\subsection{Entropy bounds}

Essential to our argument are entropic bounds related to $\mathscr{B}_{\nu, m_{*}}^{*}$. It is clear that

$$
\#\left\{D_{j}(\boldsymbol{u}):\|\boldsymbol{u}\| \leq 1\right\} \leq\left(\begin{array}{c}
d_{j} \\
2^{k} \wedge d_{j}
\end{array}\right) 2^{2^{k} \wedge d_{j}} \leq \exp \left(\left(2^{k} \wedge d_{j}\right)\left(\log 2+1+\left(\log \left(d_{j} / 2^{k}\right)\right)_{+}\right)\right),
$$

so that by (16)

$$
\left|\mathscr{B}_{m_{j}, d_{j}}\right| \leq \prod_{k=0}^{m_{j}}\left(\begin{array}{c}
d_{j} \\
2^{k} \wedge d_{j}
\end{array}\right) 2^{2^{k} \wedge d_{j}} \leq \exp \left(d_{j} \sum_{\ell=1}^{\infty} 2^{-\ell}\left(\log 2+1+\log \left(2^{\ell}\right)\right)\right) \leq \exp \left(4.78 d_{j}\right)
$$

Consequently, due to $d=d_{1}+d_{2}+d_{3}$ and $4.78 \leq 21 / 4$,

$$
\left|\mathscr{B}_{\nu, m_{*}}^{*}\right| \leq \prod_{j=1}^{3}\left|\mathscr{B}_{m_{j}, d_{j}}\right| \leq e^{(21 / 4) d} .
$$

We derive tighter entropy bounds for slices of $\mathscr{B}_{\nu, m_{*}}^{*}$ by considering

$$
\mathscr{D}_{\nu, j, k}=\left\{D_{j}(\boldsymbol{Y}): \boldsymbol{Y} \in \mathscr{B}_{\nu, m_{*}}^{*},\left\|D_{j}(\boldsymbol{Y})\right\|_{\mathrm{HS}}^{2} \leq 2^{k-j}\right\} .
$$

Here and in the sequel, we suppress the dependence of $\mathscr{D}$ on quantities such as $m_{*}, m_{1}, m_{2}, m_{3}$ for brevity, when no confusion occurs.

Lemma 12 Let $L(x, y)=\max \{1, \log (e y / x)\}$ and $\nu \geq 1$. For all $0 \leq k \leq j \leq m^{*}$,

$$
\log \left|\mathscr{D}_{\nu, j, k}\right| \leq(21 / 4) J(\nu, j, k),
$$

where $J(\nu, j, k)=(j+2) \sqrt{\nu 2^{k-1}} L\left(\sqrt{\nu 2^{k-1}},(j+2) d\right)$.

Proof of Lemma 12, We first bound the entropy of a single block. Let

$$
\begin{aligned}
& \mathscr{D}_{\nu, \ell}^{\text {(block) }}=\left\{\operatorname{sgn}\left(u_{a}\right) \operatorname{sgn}\left(v_{b}\right) \operatorname{sgn}\left(w_{c}\right) \mathbb{I}\{(a, b, c) \in A \times B \times C\}:\right. \\
& h(A \times B \times C) \leq \nu,|A||B||C|=\ell\} .
\end{aligned}
$$

By the constraints on the size and aspect ratio of the block,

$$
\max \left(|A|^{2},|B|^{2},|C|^{2}\right) \leq \nu|A||B||C| \leq \nu \ell
$$

By dividing $\mathscr{D}_{\nu, \ell}^{\text {(block) }}$ into subsets according to $\left(\ell_{1}, \ell_{2}, \ell_{3}\right)=(|A|,|B|,|C|)$, we find

$$
\left|\mathscr{D}_{\nu, \ell}^{\text {(block })}\right| \leq \sum_{\ell_{1} \ell_{2} \ell_{3}=\ell, \max \left(\ell_{1}, \ell_{2}, \ell_{3}\right) \leq \sqrt{\nu \ell}} 2^{\ell_{1}+\ell_{2}+\ell_{3}}\left(\begin{array}{c}
d_{1} \\
\ell_{1}
\end{array}\right)\left(\begin{array}{l}
d_{2} \\
\ell_{2}
\end{array}\right)\left(\begin{array}{l}
d_{3} \\
\ell_{3}
\end{array}\right)
$$


By the Stirling formula, for $i=1,2,3$,

$$
\log \left[\sqrt{2 \pi \ell_{i}} 2^{\ell_{i}}\left(\begin{array}{c}
d_{i} \\
\ell_{i}
\end{array}\right)\right] \leq \ell_{i} L\left(\ell_{i}, 2 d\right) \leq \sqrt{\nu \ell} L(\sqrt{\nu \ell}, 2 d)
$$

We note that $k(k+1) /\left(2 \sqrt{q^{k}}\right)$ is no greater than 2.66, 1.16 and 1 respectively for $q=2$, $q=3$ and $q \geq 5$. Let $\ell=\prod_{j=1}^{m} q_{j}^{k_{j}}$ with distinct prime factors $q_{j}$. We get

$$
\left|\left\{\left(\ell_{1}, \ell_{2}, \ell_{3}\right): \ell_{1} \ell_{2} \ell_{3}=\ell\right\}\right|=\prod_{j=1}^{m}\left(\begin{array}{c}
k_{j}+1 \\
2
\end{array}\right) \leq 2.66 \times 1.16 \prod_{j=1}^{m} \sqrt{q_{j}^{k_{j}}} \leq \pi \ell^{1 / 2} \leq \prod_{i=1}^{3} \sqrt{2 \pi \ell_{i}} .
$$

It follows that

$$
\left|\mathscr{D}_{\nu, \ell}^{\text {(block) }}\right| \leq \exp (3 \sqrt{\nu \ell} L(\sqrt{\nu \ell}, 2 d))
$$

Due to the constraint $i_{1}+i_{2}+i_{3}=j$ in defining $\mathscr{B}_{\nu, m_{*}}^{*}$, for any $\boldsymbol{Y} \in \mathscr{B}_{\nu, m_{*}}^{*}, D_{j}(\boldsymbol{Y})$ is composed of at most $i^{*}=\left(\begin{array}{c}j+2 \\ 2\end{array}\right)$ blocks. Since the sum of the sizes of the blocks is bounded by $2^{k}$, (23) yields

$$
\begin{aligned}
\left|\mathscr{D}_{\nu, j, k}\right| & \leq \sum_{\ell_{1}+\ldots+\ell_{i^{*}} \leq 2^{k}} \prod_{i=1}^{i^{*}}\left|\mathscr{D}_{\nu \ell_{i}}^{\text {(block) }}\right| \\
& \leq \sum_{\ell_{1}+\ldots+\ell_{i^{*}} \leq 2^{k}} \exp \left(\sum_{i=1}^{i^{*}} 3 \sqrt{\nu \ell_{i}} L\left(\sqrt{\nu \ell_{i}}, 2 d\right)\right) \\
& \leq\left(2^{k}\right)^{i^{*}} \max _{\ell_{1}+\ldots+\ell_{i^{*}} \leq 2^{k}} \exp \left(\sum_{i=1}^{i^{*}} 3 \sqrt{\nu \ell_{i}} L\left(\sqrt{\nu \ell_{i}}, 2 d\right)\right) .
\end{aligned}
$$

It follows from the definition of $L(x, y)$ and the Cauchy-Schwarz inequality that

$$
\begin{aligned}
\sum_{i=1}^{i^{*}} \sqrt{\ell_{i}} L\left(\sqrt{\nu \ell_{i}}, 2 d\right) & =\sum_{i=1}^{i^{*}} \sqrt{\ell_{i}}\left(L\left(\sqrt{\nu 2^{k}}, 2 d\right)+\log \left(\sqrt{2^{k} / \ell_{i}}\right)\right) \\
& \leq \sqrt{2^{k}}\left(\sqrt{i^{*}} L\left(\sqrt{\nu 2^{k}}, 2 d\right)+\sum_{i=1}^{i^{*}} \sqrt{\ell_{i} / 2^{k}} \log \left(\sqrt{2^{k} / \ell_{i}}\right)\right) \\
& \leq \sqrt{i^{*} 2^{k}}\left(L\left(\sqrt{\nu 2^{k}}, 2 d\right)+\log \left(\sqrt{i^{*}}\right)\right),
\end{aligned}
$$

where the last inequality above follows from the fact that subject to $u_{1}, u_{2} \geq 0$ and $u_{1}^{2}+u_{2}^{2} \leq$ $2 c^{2} \leq 2$, the maximum of $-u_{1} \log \left(u_{1}\right)-u_{2} \log \left(u_{2}\right)$ is attained at $u_{1}=u_{2}=c$. Consequently, since $i^{*} \leq\left(\begin{array}{c}j+2 \\ 2\end{array}\right)$,

$$
\log \left|\mathscr{D}_{\nu, j, k}\right| \leq i^{*} \log \left(2^{k}\right)+3 \sqrt{i^{*} \nu 2^{k}} L\left(\sqrt{\nu 2^{k}}, 2 d \sqrt{\left(\begin{array}{c}
j+2 \\
2
\end{array}\right)}\right) .
$$


We note that $j \geq k, \sqrt{2^{k}} \geq k \sqrt{8} / 3, \nu \geq 1$ and $x L(x, y)$ is increasing in $x$, so that

$$
\frac{\sqrt{i^{*} \nu 2^{k}} L\left(\sqrt{\nu 2^{k}}, 2 d \sqrt{\left(\begin{array}{c}
j+2 \\
2
\end{array}\right)}\right)}{i^{*} \log \left(2^{k}\right)} \geq \frac{\sqrt{8} / 3 L\left(k \sqrt{8} / 2,2 d \sqrt{\left.\left(\begin{array}{c}
j+2 \\
2
\end{array}\right)\right)}\right.}{\sqrt{i^{*}} \log 2} \geq \frac{\sqrt{8} \log (e d)}{\sqrt{i^{*}} \log 8} .
$$

Moreover, because $2^{m^{*}+3 / 2} \leq \sqrt{8} \prod_{j=1}^{3}\left(2 d_{j}\right) \leq \sqrt{8}(2 d / 3)^{3} \leq d^{3}$, we get $\sqrt{2 i^{*}} \leq$ $\sqrt{(j+1)(j+2)} \leq j+3 / 2 \leq m^{*}+3 / 2 \leq(3 / \log 2) \log d$, so that the right-hand side of the above inequality is no smaller than $(4 / \log 8)(\log 2) / 3=4 / 9$. It follows that

$$
\log \left|\mathscr{D}_{\nu, j, k}\right| \leq(3+9 / 4) \sqrt{i^{*} \nu 2^{k}} L\left(\sqrt{\nu 2^{k}}, 2 d \sqrt{\left(\begin{array}{c}
j+2 \\
2
\end{array}\right)}\right)
$$

This yields (22) due to $i^{*} \leq\left(\begin{array}{c}j+2 \\ 2\end{array}\right)$.

By (21), the entropy bound in Lemma 12 is useful only when $0 \leq k \leq j \leq m_{*}$, where

$$
m_{*}=\min \left\{x: x \geq m^{*} \text { or } J\left(\nu_{1}, x, x\right) \geq d\right\} .
$$

\subsection{Probability bounds}

We are now ready to derive a useful upper bound for

$$
\max _{\boldsymbol{X} \in \mathscr{U}(\eta)} \mathbb{P}\left\{\left\|\frac{d_{1} d_{2} d_{3}}{n} \sum_{i=1}^{n} \epsilon_{i} \mathcal{P}_{\left(a_{i}, b_{i}, c_{i}\right)} \boldsymbol{X}\right\| \geq t\right\}
$$

Let $\boldsymbol{X} \in \mathscr{U}(\eta)$. For brevity, write $\boldsymbol{Z}_{i}=d_{1} d_{2} d_{3} \epsilon_{i} \mathcal{P}_{\left(a_{i}, b_{i}, c_{i}\right)} \boldsymbol{X}$ and

$$
\boldsymbol{Z}=\frac{d_{1} d_{2} d_{3}}{n} \sum_{i=1}^{n} \epsilon_{i} \mathcal{P}_{\left(a_{i}, b_{i}, c_{i}\right)} \boldsymbol{X}=\frac{1}{n} \sum_{i=1}^{n} \boldsymbol{Z}_{i}
$$

Let $\Omega=\left\{\left(a_{i}, b_{i}, c_{i}\right): i \leq n\right\}$. In the light of Lemma 10, we shall proceed conditional on the event that $\nu_{\Omega} \leq \nu_{1}$ in this subsection. In this event, Lemma 11 yields

$$
\begin{aligned}
\|\boldsymbol{Z}\| & \leq 8 \max _{\boldsymbol{Y} \in \mathscr{B}_{\nu_{1}, m_{*}}^{*}}\langle\boldsymbol{Y}, \boldsymbol{Z}\rangle \\
& =8 \max _{\boldsymbol{Y} \in \mathscr{B}_{\nu_{1}, m_{*}}^{*}}\left(\sum_{0 \leq j \leq m_{*}}\left\langle D_{j}(\boldsymbol{Y}), \boldsymbol{Z}\right\rangle+\left\langle S_{*}(\boldsymbol{Y}), \boldsymbol{Z}\right\rangle\right),
\end{aligned}
$$

where $m_{*}$ is as in (24) with the given $\nu_{1}$ and $S_{*}(\boldsymbol{Y})=\sum_{j>m_{*}} D_{j}(\boldsymbol{Y})$.

Let $\boldsymbol{Y} \in \mathscr{B}_{\nu_{1}, m_{*}}^{*}$ and $\boldsymbol{Y}_{j}=D_{j}(\boldsymbol{Y})$. Recall that for $\boldsymbol{Y}_{j} \neq 0,2^{-j} \leq\left\|\boldsymbol{Y}_{j}\right\|_{\mathrm{HS}}^{2} \leq 1$, so that $\boldsymbol{Y}_{j} \in \cup_{k=0}^{j} \mathscr{D}_{\nu_{1}, j, k}$. To bound the first term on the right-hand side of (25), consider

$$
\mathbb{P}\left\{\max _{\boldsymbol{Y}_{j} \in \mathscr{D}_{\nu_{1}, j, k} \backslash \mathscr{D}_{\nu_{1}, j, k-1}}\left\langle\boldsymbol{Y}_{j}, \boldsymbol{Z}\right\rangle \geq t\left(m_{*}+2\right)^{-1 / 2}\left\|\boldsymbol{Y}_{j}\right\|_{\mathrm{HS}}\right\}
$$


with $0 \leq k \leq j \leq m_{*}$. Because $\left\|\boldsymbol{Z}_{i}\right\|_{\max } \leq d_{1} d_{2} d_{3}\|\boldsymbol{X}\|_{\max } \leq \eta \sqrt{d_{1} d_{2} d_{3}}$, for any $\boldsymbol{Y}_{j} \in \mathscr{D}_{\nu_{1}, j, k}$,

$$
\left|\left\langle\boldsymbol{Y}_{j}, \boldsymbol{Z}_{i}\right\rangle\right| \leq 2^{-j / 2} \eta \sqrt{d_{1} d_{2} d_{3}}, \quad \mathbb{E}\left\langle\boldsymbol{Y}_{j}, \boldsymbol{Z}_{i}\right\rangle^{2} \leq \eta^{2}\left\|\boldsymbol{Y}_{j}\right\|_{\mathrm{HS}}^{2} \leq \eta^{2} 2^{k-j} .
$$

Let $h_{0}(u)=(1+u) \log (1+u)-u$. By Bennet's inequality,

$$
\begin{aligned}
& \mathbb{P}\left\{\left\langle\boldsymbol{Y}_{j}, \boldsymbol{Z}\right\rangle \geq t 2^{(k-j-1) / 2}\left(m_{*}+2\right)^{-1 / 2}\right\} \\
\leq & \exp \left(-\frac{n\left(\eta^{2} 2^{k-j}\right)}{2^{-j} \eta^{2} d_{1} d_{2} d_{3}} h_{0}\left(\frac{\left(t 2^{(k-j-1) / 2}\left(m_{*}+2\right)^{-1 / 2}\right)\left(2^{-j / 2} \eta \sqrt{d_{1} d_{2} d_{3}}\right)}{\eta^{2} 2^{k-j}}\right)\right) \\
= & \exp \left(-\frac{n 2^{k}}{d_{1} d_{2} d_{3}} h_{0}\left(\frac{t \sqrt{d_{1} d_{2} d_{3}}}{\eta \sqrt{\left(m_{*}+2\right) 2^{k+1}}}\right)\right) .
\end{aligned}
$$

Recall that $\mathscr{D}_{\nu, j, k}=\left\{\boldsymbol{Y}_{j}=D_{j}(\boldsymbol{Y}): \boldsymbol{Y} \in \mathscr{B}_{\nu, m_{*}}^{*},\left\|\boldsymbol{Y}_{j}\right\|_{\mathrm{HS}}^{2} \leq 2^{k-j}\right\}$. By Lemma 12,

$$
\begin{aligned}
& \mathbb{P}\left\{\max _{\boldsymbol{Y}_{j} \in \mathscr{D}_{\nu_{1}, j, k} \backslash \mathscr{D}_{\nu_{1}, j, k-1}}\left\langle\boldsymbol{Y}_{j}, \boldsymbol{Z}\right\rangle \geq t\left(m_{*}+2\right)^{-1 / 2}\left\|\boldsymbol{Y}_{j}\right\|_{\mathrm{HS}}\right\} \\
\leq & \left|\mathscr{D}_{\nu_{1}, j, k}\right| \max _{\boldsymbol{Y}_{j} \in \mathscr{D}_{\nu_{1}, j, k}} \mathbb{P}\left\{\left\langle\boldsymbol{Y}_{j}, \boldsymbol{Z}\right\rangle \geq t 2^{(k-j-1) / 2}\left(m_{*}+2\right)^{-1 / 2}\right\} \\
\leq & \exp \left((21 / 4) J\left(\nu_{1}, j, k\right)-\frac{n 2^{k}}{d_{1} d_{2} d_{3}} h_{0}\left(\frac{t \sqrt{d_{1} d_{2} d_{3}}}{\eta \sqrt{\left(m_{*}+2\right) 2^{k+1}}}\right)\right) .
\end{aligned}
$$

Let $L_{k}=1 \vee \log \left(e d\left(m_{*}+2\right) / \sqrt{\nu_{1} 2^{k-1}}\right)$. By the definition of $J(\nu, j, k)$ in Lemma 12,

$$
J\left(\nu_{1}, j, k\right) \leq J\left(\nu_{1}, m_{*}, k\right)=\left(m_{*}+2\right) \sqrt{2^{k-1} \nu_{1}} L_{k} \leq J\left(\nu_{1}, m_{*}, m_{*}\right)=d .
$$

Let $x \geq 1$ and $t_{1}$ be a constant satisfying

$$
n t_{1} \geq 24 \eta\left(m_{*}+2\right)^{3 / 2} \sqrt{\nu_{1} d_{1} d_{2} d_{3}}, \quad n x t_{1}^{2} h_{0}(1) \geq 12 \eta^{2}\left(m_{*}+2\right)^{2} \sqrt{e} d L_{0} .
$$

We prove that for all $x \geq 1$ and $0 \leq k \leq j \leq m_{*}$

$$
\frac{n 2^{k}}{d_{1} d_{2} d_{3}} h_{0}\left(\frac{x t_{1} \sqrt{d_{1} d_{2} d_{3}}}{\eta \sqrt{\left(m_{*}+2\right) 2^{k+1}}}\right) \geq 6 x J\left(\nu_{1}, m_{*}, k\right) \geq 6 x J\left(\nu_{1}, j, k\right) .
$$

Consider the following three cases:

Case 1: $\quad \frac{x t_{1} \sqrt{d_{1} d_{2} d_{3}}}{\eta \sqrt{\left(m_{*}+2\right) 2^{k+1}}} \geq\left[\max \left\{1, \frac{e d\left(m_{*}+2\right)}{\sqrt{\nu_{1} 2^{k-1}}}\right\}\right]^{1 / 2}$,

Case 2: $\quad 1<\frac{x t_{1} \sqrt{d_{1} d_{2} d_{3}}}{\eta \sqrt{\left(m_{*}+2\right) 2^{k+1}}} \leq\left[\frac{e d\left(m_{*}+2\right)}{\sqrt{\nu_{1} 2^{k-1}}}\right]^{1 / 2}$,

Case 3: $\quad \frac{x t_{1} \sqrt{d_{1} d_{2} d_{3}}}{\eta \sqrt{\left(m_{*}+2\right) 2^{k+1}}} \leq 1$. 
Case 1: Due to $h_{0}(u) \geq(u / 2) \log (1+u)$ for $u \geq 1$ and the lower bound for $n t_{1}$,

$$
\begin{aligned}
\frac{n 2^{k}}{d_{1} d_{2} d_{3}} h_{0}\left(\frac{x t_{1} \sqrt{d_{1} d_{2} d_{3}}}{\eta \sqrt{\left(m_{*}+2\right) 2^{k+1}}}\right) & \geq \frac{n 2^{k}}{d_{1} d_{2} d_{3}}\left(\frac{x t_{1} \sqrt{d_{1} d_{2} d_{3}}}{\eta \sqrt{\left(m_{*}+2\right) 2^{k+1}}}\right) \frac{L_{k}}{4} \\
& \geq 6 x\left(m_{*}+2\right) \sqrt{\nu_{1} 2^{k-1}} L_{k} .
\end{aligned}
$$

Case 2: Due to $\left(m_{*}+2\right) \sqrt{2^{k-1} \nu_{1}} \leq d$, we have

$$
\frac{1}{\sqrt{d_{1} d_{2} d_{3}}} \geq \frac{x t_{1} \sqrt{e}\left(m_{*}+2\right)}{\eta \sqrt{\left(m_{*}+2\right) 2^{k+1}}}\left[\frac{e d\left(m_{*}+2\right)}{\sqrt{\nu_{1} 2^{k-1}}}\right]^{-1}=\frac{x t_{1} \sqrt{\nu_{1} 2^{k-1}}}{\eta d \sqrt{e\left(m_{*}+2\right) 2^{k+1}}} .
$$

Thus, due to $h_{0}(u) \geq u h_{0}(1)$ for $u \geq 1$, we have

$$
\begin{aligned}
\frac{n 2^{k}}{d_{1} d_{2} d_{3}} h_{0}\left(\frac{x t_{1} \sqrt{d_{1} d_{2} d_{3}}}{\eta \sqrt{\left(m_{*}+2\right) 2^{k+1}}}\right) & \geq\left(\frac{x t_{1} \sqrt{\nu_{1} 2^{k-1}}}{\eta d \sqrt{e\left(m_{*}+2\right) 2^{k+1}}}\right) \frac{n \sqrt{2^{k-1}} x t_{1} h_{0}(1)}{\eta \sqrt{m_{*}+2}} \\
& =\frac{n x^{2} t_{1}^{2} h_{0}(1) \sqrt{\nu_{1} 2^{k-1}}}{2 \eta^{2}\left(m_{*}+2\right) \sqrt{e} d} .
\end{aligned}
$$

Because $n x t_{1}^{2} h_{0}(1) \geq 12 \eta^{2}\left(m_{*}+2\right)^{2} \sqrt{e} d L_{0}$ and $L_{0} \geq L_{k}$, it follows that

$$
\frac{n 2^{k}}{d_{1} d_{2} d_{3}} h_{0}\left(\frac{x t_{1} \sqrt{d_{1} d_{2} d_{3}}}{\eta \sqrt{\left(m_{*}+2\right) 2^{k+1}}}\right) \geq 6 x\left(m_{*}+2\right) \sqrt{\nu_{1} 2^{k-1}} L_{k} .
$$

Case 3: Due to $h_{0}(u) \geq u^{2} h_{0}(1)$ for $0 \leq u \leq 1$ and $n x t_{1}^{2} h_{0}(1) \geq 12 \eta^{2}\left(m_{*}+2\right) d$, we have

$$
\frac{n 2^{k}}{d_{1} d_{2} d_{3}} h_{0}\left(\frac{x t_{1} \sqrt{d_{1} d_{2} d_{3}}}{\eta \sqrt{\left(m_{*}+2\right) 2^{k+1}}}\right) \geq \frac{n x^{2} t_{1}^{2} h_{0}(1)}{2 \eta^{2}\left(m_{*}+2\right)} \geq 6 x d \geq 6 x J\left(\nu_{1}, m_{*}, k\right) .
$$

Thus, (28) holds in all three cases.

It follows from (26) and (28) that for $t_{1}$ satisfying (27) and all $x \geq 1$

$$
\mathbb{P}\left\{\max _{\boldsymbol{Y}_{j} \in \mathscr{D}_{\nu_{1}, j, k} \backslash \mathscr{D}_{\nu_{1}, j, k-1}}\left\langle\boldsymbol{Y}_{j}, \boldsymbol{Z}\right\rangle \geq \frac{x t_{1}\left\|\boldsymbol{Y}_{j}\right\|_{\mathrm{HS}}}{\sqrt{m_{*}+2}}\right\} \leq \exp \left(-(6 x-21 / 4) J\left(\nu_{1}, m_{*}, k\right)\right) .
$$

We note that $J\left(\nu_{1}, m_{*}, k\right) \geq J\left(1, m_{*}, 0\right) \geq\left(m_{*}+2\right) \log \left(e d\left(m_{*}+2\right)\right)$ by the monotonicity of $x \log (y / x)$ for $x \in[1, y]$ and $\sqrt{\nu_{1} 2^{k-1}} \geq 1$. Summing over $0 \leq k \leq j \leq m_{*}$, we find by the union bound that

$$
\begin{aligned}
& \mathbb{P}\left\{\max _{0 \leq j \leq m_{*}} \max _{\boldsymbol{Y} \in \mathscr{B}_{\nu_{1}, m_{*}^{*}}^{*} D_{j}(\boldsymbol{Y}) \neq 0} \frac{\left\langle D_{j}(\boldsymbol{Y}), \boldsymbol{Z}\right\rangle}{\left\|D_{j}(\boldsymbol{Y})\right\|_{\mathrm{HS}}} \geq \frac{x t_{1}}{\left(m_{*}+2\right)^{1 / 2}}\right\} \\
\leq & \left(\begin{array}{c}
m_{*}+2 \\
2
\end{array}\right)\left\{e d\left(m_{*}+2\right)\right\}^{-(6 x-21 / 4)\left(m_{*}+2\right)}
\end{aligned}
$$

For the second term on the right-hand side of (25), we have

$$
\left|\left\langle S_{*}(\boldsymbol{Y}), \boldsymbol{Z}_{i}\right\rangle\right| \leq 2^{-m_{*} / 2} \eta \sqrt{d_{1} d_{2} d_{3}}, \quad \mathbb{E}\left(\left\langle S_{*}(\boldsymbol{Y}), \boldsymbol{Z}_{i}\right\rangle\right)^{2} \leq \eta^{2}\left\|S_{*}(\boldsymbol{Y})\right\|_{\mathrm{HS}}^{2} .
$$


As in the proof of (26) and (28), $\log \left|\mathscr{B}_{\nu_{1}, m_{*}}^{*}\right| \leq 5 d=5 J\left(\nu_{1}, m_{*}, m_{*}\right)$ implies

$$
\begin{aligned}
& \mathbb{P}\left\{\max _{\boldsymbol{Y} \in \mathscr{B}_{\nu_{1}, m_{*}}^{*}: S_{*}(\boldsymbol{Y}) \neq 0} \frac{\left\langle S_{*}(\boldsymbol{Y}), \boldsymbol{Z}\right\rangle}{\left\|S_{*}(\boldsymbol{Y})\right\|_{\mathrm{HS}}} \geq \frac{x t_{1}}{\left(m_{*}+2\right)^{1 / 2}}\right\} \\
\leq & \left(m^{*}-m_{*}\right)\left\{e d\left(m_{*}+2\right)\right\}^{-(6 x-21 / 4)\left(m_{*}+2\right)}
\end{aligned}
$$

By Cauchy Schwartz inequality, for any $\boldsymbol{Y} \in \mathscr{B}_{\nu_{1}, m_{*}}^{*}$,

$$
\frac{\left\|S_{*}(\boldsymbol{Y})\right\|_{\mathrm{HS}}+\sum_{0 \leq j \leq m_{*}}\left\|D_{j}(\boldsymbol{Y})\right\|_{\mathrm{HS}}}{\left(m_{*}+2\right)^{1 / 2}} \leq\left(\left\|S_{*}(\boldsymbol{Y})\right\|_{\mathrm{HS}}^{2}+\sum_{0 \leq j \leq m_{*}}\left\|D_{j}(\boldsymbol{Y})\right\|_{\mathrm{HS}}^{2}\right)^{1 / 2} \leq 1 .
$$

Thus, by (25), for all $t_{1}$ satisfying (27) and $x \geq 1$,

$$
\begin{aligned}
& \mathbb{P}\left\{\max _{\boldsymbol{Y} \in \mathscr{B}_{\nu_{1}, m_{*}}}\langle\boldsymbol{Y}, \boldsymbol{Z}\rangle \geq x t_{1}\right\} \\
\leq & \left\{\left(\begin{array}{c}
m_{*}+2 \\
2
\end{array}\right)+m^{*}-m_{*}\right\}\left\{\operatorname{ed}\left(m_{*}+2\right)\right\}^{-(6 x-21 / 4)\left(m_{*}+2\right)} .
\end{aligned}
$$

We now have the following probabilistic bound via (29) and Lemma 10.

Lemma 13 Let $\nu_{1}$ be as in Lemma 10, $x \geq 1, t_{1}$ as in (27) and $m_{*}$ in 24. Then,

$$
\begin{aligned}
& \max _{\boldsymbol{X} \in \mathscr{U}(\eta)} \mathbb{P}\left\{\left\|\frac{d_{1} d_{2} d_{3}}{n} \sum_{i=1}^{n} \epsilon_{i} \mathcal{P}_{\left(a_{i}, b_{i}, c_{i}\right)} \boldsymbol{X}\right\| \geq x t_{1}\right\} \\
& \leq\left\{\left(\begin{array}{c}
m_{*}+2 \\
2
\end{array}\right)+m^{*}-m_{*}\right\}\left\{\operatorname{ed}\left(m_{*}+2\right)\right\}^{-(6 x-21 / 4)\left(m_{*}+2\right)}+d^{-\beta-1} / 3 .
\end{aligned}
$$

\subsection{Proof of Lemma 7}

We are now in position to prove Lemma 7. Let $\Omega_{1}=\left\{\left(a_{i}, b_{i}, c_{i}\right), i \leq n_{1}\right\}$. By the definition of the tensor coherence $\alpha(\boldsymbol{X})$ and the conditions on $\alpha(\boldsymbol{T})$ and $\bar{r}(\boldsymbol{T})$, we have $\|\boldsymbol{W}\|_{\max } \leq$ $\eta / \sqrt{d_{1} d_{2} d_{3}}$ with $\eta=\left(\alpha_{0} \sqrt{r}\right) \wedge \sqrt{d_{1} d_{2} d_{3}}$, so that in the light of Lemmas 8, 10, and 13,

$$
\begin{aligned}
& \max _{\substack{\boldsymbol{X}: \boldsymbol{X}=\mathcal{Q}_{T} \boldsymbol{X} \\
\|\boldsymbol{X}\|_{\max } \leq\|\boldsymbol{W}\|_{\max }}} \mathbb{P}\left\{\left\|\frac{d_{1} d_{2} d_{3}}{n_{1}} \sum_{i=1}^{n_{1}} \mathcal{P}_{\left(a_{i}, b_{i}, c_{i}\right)} \boldsymbol{X}-\boldsymbol{X}\right\| \geq \frac{1}{8}\right\} \\
\leq & 4 \exp \left(-\frac{n_{1}(1 / 16)^{2} / 2}{\eta^{2}+(2 / 3) \eta(1 / 16) \sqrt{d_{1} d_{2} d_{3}}}\right) \\
& +\left\{\left(\begin{array}{c}
m_{*}+2 \\
2
\end{array}\right)+m^{*}-m_{*}\right\}\left\{e d\left(m_{*}+2\right)\right\}^{-(6 x-21 / 4)\left(m_{*}+2\right)}+d^{-\beta-1} / 3
\end{aligned}
$$

with $t=1 / 8$ in Lemma 8 and the $\nu_{1}$ in Lemma 10, provided that $x t_{1} \leq 1 / 16$ and

$$
n_{1} t_{1} \geq 24 \eta\left(m_{*}+2\right)^{3 / 2} \sqrt{\nu_{1} d_{1} d_{2} d_{3}}, \quad n_{1} x t_{1}^{2} h_{0}(1) \geq 12 \eta^{2}\left(m_{*}+2\right)^{2} \sqrt{e} d L_{0} .
$$


Thus, the right-hand side of (30) is no greater than $d^{-\beta-1}$ for certain $x>1$ and $t_{1}$ satisfying these conditions when

$$
\begin{aligned}
n_{1} \geq & c_{1}^{\prime}\left(1+\frac{1+\beta}{m_{*}+2}\right)\left(\eta \sqrt{\left(m_{*}+2\right)^{3} \nu_{1} d_{1} d_{2} d_{3}}+\eta^{2}\left(m_{*}+2\right)^{2} L_{0} d\right) \\
& +c_{1}^{\prime}(1+\beta)(\log d)\left(\eta^{2}+\eta \sqrt{d_{1} d_{2} d_{3}}\right)
\end{aligned}
$$

for a sufficiently large constant $c_{1}^{\prime}$. Because $\sqrt{2^{m_{*}-1}} \leq J\left(\nu_{1}, m_{*}, m_{*}\right)=d$, it suffices to have

$$
n_{1} \geq c_{1}^{\prime \prime}\left[(\beta+\log d)\left\{\eta \sqrt{(\log d) \nu_{1} d_{1} d_{2} d_{3}}+\eta^{2}(\log d)^{2} d\right\}+(1+\beta)(\log d) \eta \sqrt{d_{1} d_{2} d_{3}}\right] .
$$

When the sample size is $n_{1}, \nu_{1}=\left(d^{\delta_{1}} e n_{1} \max \left(d_{1}, d_{2}, d_{3}\right) /\left(d_{1} d_{2} d_{3}\right)\right) \vee\left\{(3+\beta) / \delta_{1}\right\}$ in Lemma 10 with $\delta_{1} \in[1 / \log d, 1]$. When $\nu_{1}=d^{\delta_{1}} e n_{1} \max \left(d_{1}, d_{2}, d_{3}\right) /\left(d_{1} d_{2} d_{3}\right), n_{1} \geq x \sqrt{\nu_{1} d_{1} d_{2} d_{3}}$ iff $n_{1} \geq x^{2} d^{\delta_{1}} e \max \left(d_{1}, d_{2}, d_{3}\right)$. Thus, it suffices to have

$$
\begin{aligned}
n_{1} \geq & c_{1}^{\prime \prime}\left[(\beta+\log d)^{2} \eta^{2}(\log d) d^{1+\delta_{1}}+(\beta+\log d) \eta \sqrt{(\log d)(3+\beta) \delta_{1}^{-1} d_{1} d_{2} d_{3}}\right] \\
& +c_{1}^{\prime \prime}\left[(\beta+\log d) \eta^{2}(\log d)^{2} d+(1+\beta)(\log d) \eta \sqrt{d_{1} d_{2} d_{3}}\right] .
\end{aligned}
$$

Due to $\sqrt{(1+\beta)(\log d)} \leq 1+\beta+\log d$, the quantities in the second line in the above inequality is absorbed into those in the first line. Consequently, with $\eta=\alpha_{0} \sqrt{r}$, the stated sample size is sufficient.

\section{Discussions}

In this paper, we study the performance of nuclear norm minimization in recovering a large tensor with low Tucker ranks. Our results demonstrate the benefits of not treating tensors as matrices despite its popularity.

Throughout the paper, we have focused primarily on third order tensors. In principle, our technique can also be used to treat higher order tensors although the analysis is much more tedious and the results quickly become hard to describe. Here we outline a considerably simpler strategy which yields similar sample size requirement as the vanilla nuclear norm minimization. The goal is to illustrate some unique and interesting phenomena associated with higher order tensors.

The idea is similar to matricization - instead of unfolding a $N$ th order tensor into a matrix, we unfold it into a cubic or nearly cubic third order tensor. To fix ideas, we shall restrict our attention to hyper cubic $N$ th order tensors with $d_{1}=\ldots=d_{N}=$ : $d$ and $r_{1}(\boldsymbol{T}), r_{2}(\boldsymbol{T}), \ldots, r_{N}(\boldsymbol{T})$ are bounded from above by a constant. The discussion can be straightforwardly extended to more general situations. In this case, the resulting third order 
tensor will have dimensions either $d^{\lfloor N / 3\rfloor}$ or $d^{\lfloor N / 3\rfloor+1}$, and Tucker ranks again bounded. Here $\lfloor x\rfloor$ stands for the integer part of $x$. Our results on third order tensor then suggests a sample size requirement of

$$
n \asymp d^{N / 2} \operatorname{polylog}(d) .
$$

This is to be compared with a matricization approach that unfolds an $N$ th order tensor to a (nearly) square matrix (see, e.g., Mu et al., 2013) - where the sample size requirement is $d^{\lceil N / 2\rceil} \operatorname{polylog}(d)$. It is interesting to notice that, in this special case, unfolding a higher order tensor to a third order tensor is preferable to matricization when $N$ is odd.

\section{References}

Candès, E.J. and Recht, B. (2008), Exact matrix completion via convex optimization, Foundations of Computational Mathematics, 9, 717-772.

Candès, E.J. and Tao, T. (2009), The power of convex relaxation: Near-optimal matrix completion, IEEE Transactions on Information Theory, 56(5), 2053-2080.

Gandy, S., Recht, B. and Yamada, I. (2011), Tensor completion and low-n-rank tensor recovery via convex optimization, Inverse Problems, 27(2), 025010.

Gross, D. (2011), Recovering low-rank matrices from few coefficients in any basis, IEEE Transaction on Information Theory, 57, 1548-1566.

Hillar, C. and Lim, L.H. (2013), Most tensor problems are NP-hard, Journal of the ACM, 60(6), Art. 45.

Kolda, T.G. and Bader, B.W. (2009), Tensor decompositions and applications, SIAM Review, 51(3), 455-500.

Kruskal, J. B. (1989), Rank, decomposition, and uniqueness for 3-way and N-way arrays, in "Multiway data analysis", North-Holland, Amsterdam, pp. 7-18.

Li, N. and Li, B. (2010), Tensor completion for on-board compression of hyperspectral images, In Image Processing (ICIP), 2010 17th IEEE International Conference on, 517520.

Liu, J., Musialski, P., Wonka, P. and Ye, J. (2009), Tensor completion for estimating missing values in visual data, In $I C C V, 2114-2121$.

Mu, C., Huang, B., Wright, J. and Goldfarb, D. (2013), Square deal: lower bounds and improved relaxations for tensor recovery, arXiv: 1307.5870. 
Recht, B. (2011), A simpler approach to matrix completion, Journal of Machine Learning Research, 12, 3413-3430.

Semerci, O., Hao, N., Kilmer, M. and Miller, E. (2013), Tensor based formulation and nuclear norm regularizatin for multienergy computed tomography, to appear in IEEE Transactions on Image Processing.

Sidiropoulos N.D. and Nion, N. (2010), Tensor algebra and multi-dimensional harmonic retrieval in signal processing for mimo radar, IEEE Trans. on Signal Processing, 58(11), 5693-5705.

Signoretto, M., De Lathauwer, L. and Suykens, J. (2010), Nuclear norms for tensors and their use for convex multilinear estimation.

Signoretto, M., Van de Plas, R., De Moor, B. and Suykens, J. (2011), Tensor versus matrix completion: A comparison with application to spectral data, IEEE SPL, 18(7), 403-406.

Tomioka, R., Hayashi, K. and Kashima, H. (2010), Estimation of low-rank tensors via convex optimization, arXiv preprint arXiv:1010.0789.

Tomioka, R., Suzuki, T., Hayashi, K. and Kashima, H. (2011), Statistical performance of convex tensor decomposition, Advances in Neural Information Processing Systems (NIPS), 137.

Tropp, J. (2012), User-friendly tail bounds for sums of random matrices, Foundations of Computational Mathematics, 12, 389-434.

Watson, G. A. (1992), Characterization of the subdifferential of some matrix norms. Linear Algebra Appl., 170, 33-45. 